SERVIÇO DE PÓS-GRADUAÇÃO DO ICMC-USP

Data de Depósito: 15/01/2004

Assinatura:

\title{
Singularidades das superfícies regradas em $\mathbb{R}^{3}$
}

Rodrigo Martins ${ }^{1}$

Orientador: Profa. Dra. Maria Aparecida Soares Ruas

Dissertação apresentada ao Instituto de Ciências Matemáticas e de Computação da Universidade de São Paulo, como parte dos requisitos para obtenção do título de Mestre em Matemática.

\section{USP - São Carlos}

Janeiro/2004

\footnotetext{
${ }^{1}$ Este trabalho teve suporte financeiro da Fapesp proc: 01/11292-5
} 


\begin{abstract}
We study generic singularities of ruled surfaces in $\mathbb{R}^{3}$. In this work we show that generic singularities appearing in the set of ruled surfaces are the same that occur in the set of map germs from $\mathbb{R}^{2}$ to $\mathbb{R}^{3}$, while the generic singularities of developable surfaces are more degenerate.
\end{abstract}




\section{Resumo}

Estudaremos as singularidades genéricas de superfícies regradas em $\mathbb{R}^{3}$. O objetivo do trabalho é mostrar que as singularidades genéricas que ocorrem no conjunto das superfícies regradas são as mesmas que ocorrem no conjunto das aplicações diferenciáveis de $\mathbb{R}^{2} \mathrm{em}$ $\mathbb{R}^{3}$, enquanto que as singularidades genéricas das superfícies desenvolvíveis, que formam um subconjunto das superfícies regradas, são mais degeneradas. 
$A$ minha mãe $e^{a}$, que batalhou a vida toda para que eu chegasse até aqui.

${ }^{a}$ Creuza M. Martins, 08/11/1948 à 01/04/1996. 


\section{Agradecimentos}

Acima de tudo a Deus, que foi e é meu grande companheiro, que esteve comigo até aqui e que nunca me deixou fraquejar nem desistir. "DEUS a ti toda Honra, o Poder e a Glória pelos séculos dos séculos".

À Prof. Maria Ap. Soares Ruas, pela orientação, pela amizade e por ter sido tão importante para o meu crescimento profissional.

À toda minha família, pai, irmã, tios, tias, primos e amigos (que fazem parte da família) que colaboram muito para que esta etapa fosse concluída.

Especialmente a minha irmã, Roseli que dispensou suas orações e ações para meu fortalecimento espiritual e físico durante toda a caminhada.

Aos meus amigos da graduação e da pós-graduação, que me ajudaram de alguma forma a ultrapassar muitos obstáculos e tornaram esta caminhada mais agradável. E a meus amigos da Igreja Metodista de Penápolis que sempre guardaram meu lugar com eles enquanto estive fora, estudando.

A todos da E.E.P.S.G. Profa. Maria M. C. Castilhos de Glicério/SP, que ajudaram a moldar quem sou. Aos professores da UNESP de Rio Claro, principalmente as professoras Rosa e Suzi por terem sido mais que professoras, grandes amigas e as vezes até mãezonas. De maneira muito especial a professora Alice K. M. Libardi que é a peça fundamental da minha formação acadêmica e por ter puxado minha orelha quando foi necessário e ter me dado a maior força quando precisei. Valeu Alice !!!!!!!

Enfim, a todos aqueles que colaboraram de alguma forma para a realização deste trabalho. 


\section{Sumário}

Introdução $\quad$ V

1 Superfícies Regradas 1

1.1 Superfícies Regradas . . . . . . . . . . . . . . . . . . 1

1.2 Curva de Estrição . . . . . . . . . . . . . . . . . . . . . . . . . . . 2

1.3 Superfícies Desenvolvíveis . . . . . . . . . . . . . . . 6

2 Teoria de Singularidades $\quad 9$

2.1 Germes e Jatos . . . . . . . . . . . . . . . . . . . . 9

2.2 A topologia $C^{\infty}(X, Y)$ de Whitney . . . . . . . . . . . . . 10

2.3 Ação de Grupos de Lie em Variedades . . . . . . . . . . . . . . . . . 11

2.4 Transversalidade . . . . . . . . . . . . . . . . . . 11

2.5 Genericidade e Estabilidade . . . . . . . . . . . . . . . . . . 12

2.5.1 Estabilidade . . . . . . . . . . . . . . . . . 13

2.6 Conjunto de Singularidades de $\mathbf{1}^{\mathrm{a}}$ Ordem . . . . . . . . . . . . . . 14

$2.7 \mathcal{A}$-equivalência e $\mathcal{K}$-equivalência . . . . . . . . . . . . . . . . . . . 14

2.8 Determinação Finita e Transversal Completa . . . . . . . . . . . . 16

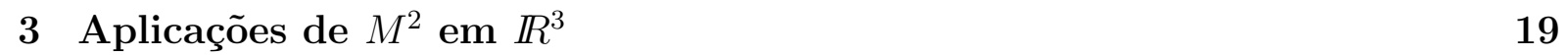

3.1 Caracterização de Aplicações Estáveis . . . . . . . . . . . . . . 19

4 Singularidades estáveis de superfícies regradas $\quad 25$

4.1 Desdobramentos ...................... . . 25

4.2 Classificação Genérica . . . . . . . . . . . . . . . . . . . 32

5 Singularidades das Superfícies Desenvolvíveis 37

5.1 Singularidade das Superfícies Tangentes . . . . . . . . . . . . . 37

5.2 Genericidade em Superfícies Tangentes . . . . . . . . . . . . . . . 39 
Referências Bibliográficas 


\section{Lista de Figuras}

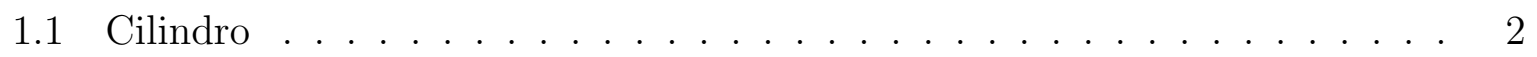

1.2 Cilindro regrado . . . . . . . . . . . . . . . . . . 2

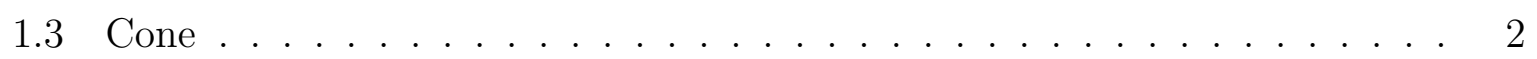

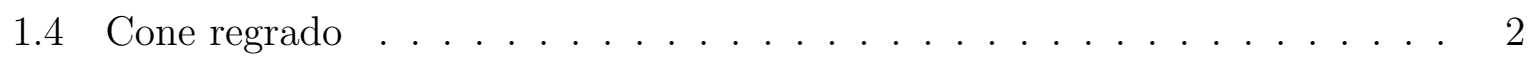

1.5 Hiperbolóide . . . . . . . . . . . . . . . . . . . 2

1.6 Hiperbolóide regrado . . . . . . . . . . . . . . . . . . . . . . . . 2

1.7 Parabolóide hiperbólico . . . . . . . . . . . . . . . . . . 5

1.8 Parabolóide hiperbólico regrado . . . . . . . . . . . . . . . . 5

1.9 Guarda-chuva de Whitney . . . . . . . . . . . . . . . . . 6

1.10 Guarda-chuva de Whitney regrado . . . . . . . . . . . . . . . 6

5.1 Imersão cuspidal . . . . . . . . . . . . . . . . . . . . . 39

5.2 Imersão cuspidal regrado . . . . . . . . . . . . . . . . . . . . . 39

5.3 Guarda-chuva de Whitney cuspidal . . . . . . . . . . . . . . . . 39

5.4 Guarda-chuva de Whitney cuspidal regrado . . . . . . . . . . . . . . . 39 



\section{Introdução}

O estudo de superfícies regradas em $\mathbb{R}^{3}$ é um assunto clássico em Geometria Diferencial. O interesse pelo tema tem reaparecido em anos recentes em diferentes áreas tais como Geometria Diferencial Projetiva ([24]), Computação Gráfica e Desenho Industrial $([10,25])$. Em geral, superfícies regradas têm singularidades e as singularidades estáveis destas superfícies foram estudadas por S. Izumiya em [12]. As superfícies desenvolvíveis são exemplos de superfícies regradas. Tais superfícies são superfícies regradas que têm curvatura Gaussiana nula em seus pontos regulares. O objetivo deste trabalho é estudar as singularidades genéricas de superfícies regradas e comparar o comportamento genérico destas singularidades com as singularidades genéricas da subclasse das superfícies desenvolvíveis.

O conjunto das superfícies regradas é um subconjunto muito pequeno no espaço $C^{\infty}\left(\mathbb{R}^{2}, \mathbb{R}^{3}\right)$ de todas as superfícies parametrizadas, entretanto mostramos que as singularidades genéricas que ocorrem no conjunto das superfícies regradas são as mesmas

que ocorrem no conjunto todo. É um fato conhecido que a única singularidade genérica que ocorre no conjunto dos germes de superfícies é o guarda-chuva de Whitney.

As singularidades que ocorrem em geral para superfícies desenvolvíveis são mais degeneradas que aquelas que ocorrem em geral na classe das superfícies regradas $([4,11,22])$.

O capítulo 1 contém as definições e resultados básicos referentes às superfícies regradas, destacando-se a importância da curva de estrição que é exatamente onde se concentram as singularidades da superfície regrada.

O capítulo 2 tem o objetivo de dar uma idéia geral da teoria de singularidades e descrever, através de teoremas como o da transversal completa, os métodos que serão usados para o estudo das singularidades genéricas das superfícies regradas.

O capítulo 3 prepara condições para o desenvolvimento do capítulo 4, fazendo um estudo das singularidades estáveis de $M^{2}$ em $\mathbb{R}^{3}$.

O capítulo 4 apresenta o tema central do trabalho, que é a caracterização das singularidades genéricas das superfícies regradas. Com base no artigo "Singularities of ruled 
surfaces in $\mathbb{R}^{3}$ ", de S. Izumiya [12], mostramos no Teorema 4.2.3 que existe um conjunto aberto e denso de superfícies regradas cujas únicas singularidades são os guarda-chuvas de Whitney.

O capítulo 5 mostra que as singularidades que ocorrem genericamente no conjunto das superfícies desenvolvíveis são singularidades mais degeneradas que o guarda-chuva de Whitney. 


\section{Capítulo 1}

\section{Superfícies Regradas}

Neste capítulo apresentamos os tópicos preliminares ao estudo das singularidades das superfícies regradas em $\mathbb{R}^{3}$ dando as definições e os resultados, relevantes sobre as superfícies regradas. As principais referências para este estudo são [3] e [12].

\subsection{Superfícies Regradas}

Definição 1.1.1 Uma superfície regrada em $\mathbb{R}^{3}$ é a imagem de uma aplicação $F: I \times J \rightarrow$ $\mathbb{R}^{3}$ definida por $F(t, v)=\alpha(t)+v \omega(t)$, onde $\alpha: I \rightarrow \mathbb{R}^{3}$ e $\omega: I \rightarrow S^{2}$ são aplicações diferenciáveis e $I$, $J$ são intervalos abertos com I limitado. Uma superfície regrada desta forma diz-se gerada pela família de curvas $\{\alpha(t), \omega(t)\}$.

Definição 1.1.2 Sejam $\{\alpha(t), \omega(t)\}$ uma família de curvas e a superfície regrada gerada por ela $\chi(t, v)=\alpha(t)+v \omega(t)$. Então $\alpha$ é chamada curva base $e \omega$ diretriz, as retas $L_{t}(v)=\alpha(t)+v \omega(t)$ são as geratrizes da superfície $\chi$.

Observação 1.1.3 Dada uma superfície $\chi$ nas condições acima seus pontos singulares são dados por $\chi_{t} \wedge \chi_{v}=0$.

Exemplo 1.1.4 Como exemplos de superfícies regradas podemos citar cilindros e cones e superfícies tangentes a uma curva regular.

Um cilindro é uma superfície regrada gerada pela família de curvas $\{\alpha, \omega\}, t \in I$ onde $\alpha(I)$ está contido num plano $P$ e $\omega(t)$ é paralelo a uma direção fixa em $\mathbb{R}^{3}$. 


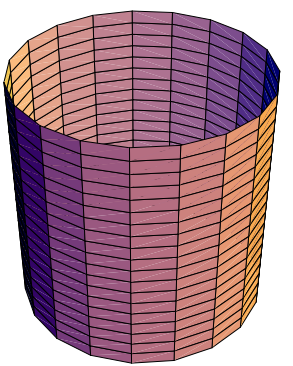

Figura 1.1: Cilindro

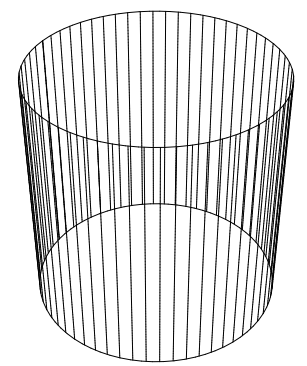

Figura 1.2: Cilindro regrado

Um cone é uma superfície regrada gerada pela família $\{\alpha, \omega\}, t \in I$ onde $\alpha(I) \subset P$ e as geratrizes passam todas por um ponto $p \notin P$.

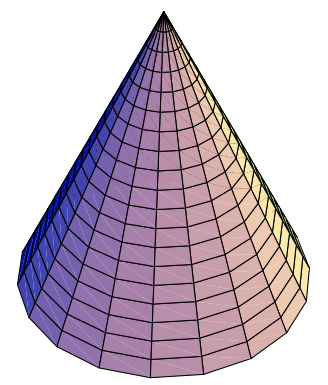

Figura 1.3: Cone

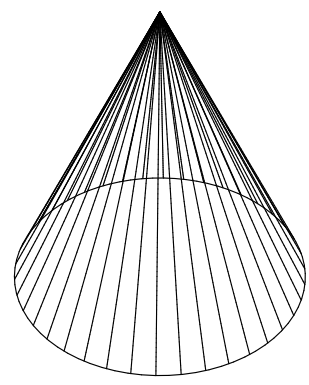

Figura 1.4: Cone regrado

Uma superfície tangente a uma curva regular é uma superfície regrada gerada pela família de curvas $\left\{\alpha, \alpha^{\prime}\right\}$ onde $\alpha$ é uma curva regular.

Exemplo 1.1.5 Seja $S^{1}$ o círculo $x^{2}+y^{2}=1$ no plano xy e seja $\alpha(s)$ uma parametrização de $S^{1}$ por comprimento de arco. Para cada $s$ definimos $\omega(s)=\alpha^{\prime}(s)+e_{3}$, onde e é $e_{0}$ vetor unitário do eixo z. Então $\chi(s, v)=\alpha(s)+v \omega(s)$ é uma superfície regrada.

Podemos colocar $\chi$ numa forma mais familiar, $\operatorname{com} \alpha(s)=(\cos (s), \sin (s), 0)$ assim

$$
\chi(s, v)=(\cos (s)-v \sin (s), \sin (s)+v \cos (s), v) .
$$

Desta forma $\chi$ é solução de $x^{2}+y^{2}-z^{2}=1$, logo $\chi$ é o traço de um hiperbolóide de revolução.

Se tomarmos $\omega(s)=-\alpha^{\prime}(s)+e_{3}$ obteremos a mesma superfície. Isto mostra que o hiperbolóide de revolução tem ao mesmo tempo dois conjuntos distintos de geratrizes. 


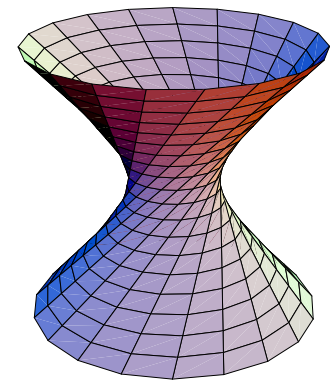

Figura 1.5: Hiperbolóide

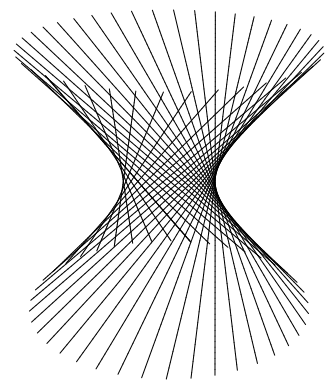

Figura 1.6: Hiperbolóide regrado

\subsection{Curva de Estrição}

Assumiremos aqui que $\omega^{\prime}(t) \neq 0$ para $t \in I$ e neste caso diremos que a superfície regrada é não cilíndrica. No caso em que os zeros de $\omega$ são isolados podemos dividir nossa superfície em pedaços e aplicar a teoria para cada pedaço. Se o conjunto dos zeros de $\omega$ contém pontos de acumulação devemos proceder com muito mais cautela. Assumiremos também que $\left\|\omega^{\prime}(t)\right\|=1$ assim estaremos assumindo que $<\omega(t), \omega^{\prime}(t)>=0$ para todo $t \in I$.

Cabe aqui uma observação, de modo geral uma superfície regrada não é gerada de maneira única pela família de curvas $\{\alpha, \omega\}$. Basta observar o exemplo do cilindro cuja curva base é uma parametrização de $S^{1}$ que sabemos não ser única. Tendo em vista esta observação podemos perguntar se existe uma parametrização que evidencie as propriedades da superfície ou simplifique os cálculos.

Queremos encontrar uma curva parametrizada $\beta$ tal que $<\beta^{\prime}(t), \omega^{\prime}(t)>=0$ para todo $t \in I$ e $\beta$ pertença a superfície regrada, isto é,

$$
\beta(t)=\alpha(t)+u(t) \omega(t)
$$

Assim se $\beta$ existe, temos que $\beta^{\prime}(t)=\alpha^{\prime}(t)+u^{\prime}(t) \omega(t)+\omega^{\prime}(t) u(t)$, portanto, como $<\omega, \omega^{\prime}>=0$, temos

$$
0=<\beta^{\prime}, \omega^{\prime}>=<\alpha^{\prime}, \omega^{\prime}>+u<\omega^{\prime}, \omega^{\prime}>
$$

$\log 0(t)=-\frac{\left.<\alpha^{\prime}(t), \omega^{\prime}(t)\right\rangle}{\left.<\omega^{\prime}(t), \omega^{\prime}(t)\right\rangle}$.

Definição 1.2.1 $\beta$ assim definida é chamada curva de estrição e seus pontos são chamados pontos centrais da superfície regrada.

Proposição 1.2.2 $\beta$ não depende da escolha da curva base $\alpha$. 
Demonstração: : De fato, seja $\bar{\alpha}$ uma outra curva base. Assim

$$
\chi(t, v)=\alpha(t)+v \omega(t)=\bar{\alpha}(t)+s \omega(t) .
$$

$\operatorname{Logo} \beta-\bar{\beta}=(\alpha-\bar{\alpha})+\frac{<\bar{\alpha}^{\prime}-\alpha^{\prime}>}{\left.<\omega^{\prime}, \omega^{\prime}\right\rangle} \omega$, entretanto $\alpha-\bar{\alpha}=(s-v) \omega$. Portanto

$$
\beta-\bar{\beta}=\left\{(s-v)+\frac{<(v-s) \omega^{\prime}, \omega^{\prime}>}{<\omega^{\prime}, \omega^{\prime}>}\right\} \omega=0
$$

A partir de agora estaremos interessados em descobrir as propriedades da curva de estrição e saber se esta escolha de curva base nos traz alguma vantagem. Assim tomaremos a superfície regrada por

$$
\chi(t, u)=\beta(t)+u \omega(t)
$$

Com esta escolha temos $\chi_{t}=\beta^{\prime}+u \omega^{\prime}, \chi_{u}=\omega$ e $\chi_{t} \wedge \chi_{u}=\beta^{\prime} \wedge \omega+u \omega^{\prime} \wedge \omega$. Como $<\omega^{\prime}, \omega>=0$ e $<\omega^{\prime}, \beta^{\prime}>=0$ concluímos que $\beta^{\prime} \wedge \omega=\lambda \omega^{\prime}$ para alguma função $\lambda$ de $t$.

Portanto $\left\|\chi_{t} \wedge \chi_{u}\right\|^{2}=\left\|\lambda \omega^{\prime}+u \omega^{\prime} \wedge \omega\right\|^{2}=\lambda^{2}\left\|\omega^{\prime}\right\|^{2}+u^{2}\left\|\omega^{\prime}\right\|^{2}=\left(\lambda^{2}+u^{2}\right)\left\|\omega^{\prime}\right\|^{2}$, disto segue que os únicos pontos singulares da superfície regrada estão sobre a curva de estrição, isto é, $u=0$ e eles ocorrem se, e somente se, $\lambda=0$.

Observemos, agora, que $\beta^{\prime} \wedge \omega=\lambda \omega^{\prime}$ portanto

$$
\lambda(t)=\frac{<\beta^{\prime}(t) \wedge \omega(t), \omega^{\prime}(t)>}{\left\|\omega^{\prime}(t)\right\|^{2}} .
$$

Indiquemos por $<\beta^{\prime} \wedge \omega, \omega^{\prime}>=\left(\beta^{\prime}, \omega, \omega^{\prime}\right)$ o produto misto dos vetores $\beta^{\prime}, \omega$ e $\omega^{\prime}$.

Calculemos a curvatura Gaussiana da superfície regrada em seus pontos regulares:

$$
\chi_{t t}=\beta^{\prime \prime}+u \omega^{\prime \prime} ; \quad \chi_{t u}=\omega^{\prime} ; \quad \chi_{u u}=0 .
$$

Os coeficientes da segunda forma fundamental são

$$
g=0 \quad \text { e } \quad f=\frac{\left(\chi_{t}, \chi_{u}, \chi_{u t}\right)}{\left\|\chi_{t} \wedge \chi_{u}\right\|}=\frac{\left(\beta^{\prime}, \omega, \omega^{\prime}\right)}{\left\|\chi_{t} \wedge \chi_{u}\right\|},
$$

$\log \mathrm{O}$

$$
K=-\frac{\lambda^{2}}{\left(\lambda^{2}+u^{2}\right)^{2}} .
$$

Isto mostra que, em pontos regulares, a curvatura $K$ de uma superfície regrada satisfaz $K \leq 0$ e $K=0$ somente ao longo das geratrizes que encontram a curva de estrição em um ponto singular.

A equação da curvatura nos permite dar uma interpretação geométrica dos pontos (regulares) centrais de uma superfície regrada. Realmente, os pontos de uma geratriz, 
exceto talvez o ponto central, são pontos regulares da superfície. Se $\lambda \neq 0$ a função $K(u)$ é uma função diferenciável sobre a geratriz e o ponto central é um ponto de máximo da função $K(u)$.

Observamos ainda que a curvatura $K$ assume os mesmos valores em pontos da geratriz que são simétricos relativamente aos pontos centrais.

Definição 1.2.3 A função $\lambda(t)$ é chamada de função distribuição dos parâmetros de $\chi$.

Observação 1.2.4 Como a curva de estrição independe da escolha da curva base, segue que o mesmo vale para a função distribuição.

Se $\chi$ é regular temos a seguinte interpretação de $\lambda$ : o vetor normal à superfície no ponto $(t, u)$ é

$$
N(t, u)=\frac{\chi_{t} \wedge \chi_{u}}{\left\|\chi_{t} \wedge \chi_{u}\right\|}=\frac{\lambda \omega^{\prime}+u \omega^{\prime} \wedge \omega}{\sqrt{\lambda^{2}+u^{2}}\left\|\omega^{\prime}\right\|},
$$

por outro lado, se $\lambda \neq 0$ temos

$$
N(t, 0)=\frac{\lambda}{|\lambda|} \frac{\omega^{\prime}}{\left\|\omega^{\prime}\right\|} .
$$

Entretanto, se $\theta$ é o ângulo formado por $N(t, u)$ e $N(t, 0)$ vale

$$
\tan \theta=\frac{|u|}{\lambda}
$$

Assim se $\theta$ é o ângulo formado pelo vetor normal em um ponto de uma geratriz e pelo vetor normal no ponto central desta geratriz, então tan $\theta$ é proporcional à distância entre os dois pontos e o coeficiente de proporcionalidade é o inverso do parâmetro de distribuição.

Exemplo 1.2.5 Consideremos $S$ o parabolóide hiperbólico $z=k x y$ com $k \neq 0$.

Para mostrar que $S$ é uma superfície regrada, observemos que a família de retas $\left(t, \frac{z}{t k}, z\right)$ para $t \neq 0$ pertencem a $S$. Se tomarmos a intersecção desta família de retas com o plano $z=0$ obtemos a curva $(t, 0,0)$. Tomando esta curva como curva base e a diretriz dada por $\omega(t)=\frac{w(t)}{|w(t)|}$ onde $w(t)=\left(0, \frac{1}{k}, t\right)$ temos

$$
\chi(t, v)=\alpha(t)+v \omega(t)=\left(t, \frac{v}{\sqrt{1+k^{2} t^{2}}}, \frac{v k t}{\sqrt{1+k^{2} t^{2}}}\right) .
$$

Como $\alpha^{\prime}(t)=(1,0,0)$, temos que a curva de estrição é mesmo $\alpha$. O parâmetro de distribuição é $\lambda=\frac{1}{k}$. Observamos também que a tangente do ângulo entre $\omega(t)$ e $\omega(0)$ é $\tan \theta=t k$.

A superfície $\chi$ é exatamente o parabolóide hiperbólico $z=k x y$. 


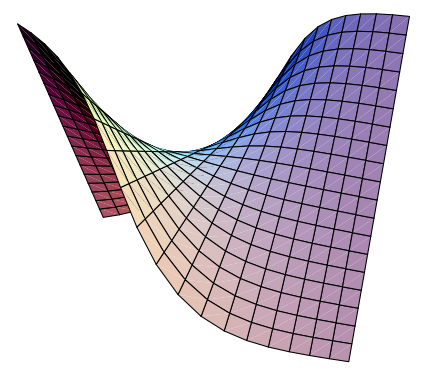

Figura 1.7: Parabolóide hiperbólico

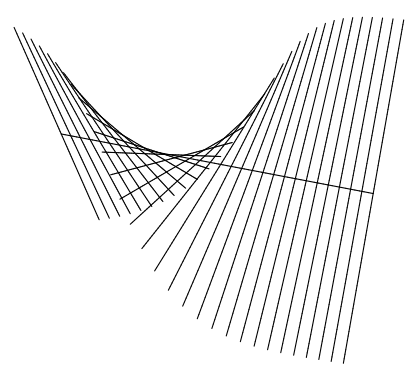

Figura 1.8: Parabolóide hiperbólico regrado

Exemplo 1.2.6 O guarda-chuva de Whitney (ou cross cap) é a imagem da aplicação $f: I \times J \rightarrow \mathbb{R}^{3}$ definida por $f(x, y)=\left(x^{2}, y, x y\right)$ onde $I$ e $J$ são intervalos de $\mathbb{R}$ contendo a origem.

Dadas as curvas $\beta(t)=\left(t^{2}, 0,0\right)$ e $\omega(t)=\left(0, \frac{1}{\sqrt{1+t^{2}}}, \frac{t}{\sqrt{1+t^{2}}}\right)$ podemos definir a superfície regrada

$$
F(t, u)=\left(t^{2}, 0,0\right)+u\left(0, \frac{1}{\sqrt{1+t^{2}}}, \frac{t}{\sqrt{1+t^{2}}}\right)
$$

com curva base $\beta$ e diretriz $\omega$. Se considerarmos a mudança de variáveis dada por

$$
\left\{\begin{array}{l}
x=t \\
y=\frac{u}{\sqrt{1+t^{2}}}
\end{array}\right.
$$

teremos $f(x(t, u), y(t, u))=F(t, u)$, assim mostramos que o cross cap é uma superfície regrada dada pelas curvas $\beta$ e $\omega$.

Como $<\beta^{\prime}(t), \omega^{\prime}(t)>=0$ para todo $t$, temos por definição que a curva $\beta$ é a curva de estrição do cross cap $F$. Como vimos, as singularidades de uma superfície regrada estão sobre a curva de estrição. Calculemos agora o parâmetro de distribuição $\lambda$.

$$
\lambda(t)=\frac{<\beta^{\prime}(t) \wedge \omega(t), \omega^{\prime}(t)>}{\left\|\omega^{\prime}(t)\right\|^{2}}=\frac{-4 t^{2}}{\left(1+t^{2}\right)\left\|\omega^{\prime 2}\right\|}
$$

Assim $\lambda(t)=0$ se, e somente se, $t=0$ e o único ponto singular da superfícies está em $(0,0,0)$. 


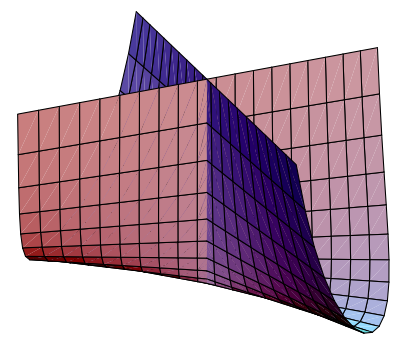

Figura 1.9: Guarda-chuva de Whitney

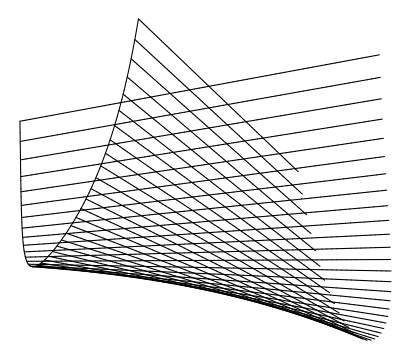

Figura 1.10: Guarda-chuva de Whitney regrado

\subsection{Superfícies Desenvolvíveis}

As superfícies desenvolvíveis constituem uma classe especial do conjunto das superfícies regradas.

Definição 1.3.1 Seja $\chi(t, v)=\alpha(t)+v \omega(t)$ uma superfície regrada qualquer (não necessariamente não cilíndrica), gerada pela familia $\{\alpha(t), \omega(t)\}$ com $\|\omega(t)\|=1$. A superfície $\chi$ é desenvolvível se $\left(\omega, \omega^{\prime}, \alpha^{\prime}\right) \equiv 0$.

Para dar uma interpretação geométrica para $\left(\omega, \omega^{\prime}, \alpha^{\prime}\right) \equiv 0$, calculemos a curvatura Gaussiana de uma superfície regrada desenvolvível em um ponto regular. Obtemos:

$$
g=0 \quad \text { e } \quad f=\frac{\left(\omega, \omega^{\prime}, \alpha^{\prime}\right)}{\left\|\chi_{t} \wedge \chi_{v}\right\|}=0,
$$

assim

$$
K=\frac{e g-f^{2}}{E G-F^{2}} \equiv 0
$$

Portanto, em pontos regulares, a curvatura Gaussiana de uma superfície regrada desenvolvível é nula.

Outro fato facilmente verificável é que, numa superfície desenvolvível, o plano tangente ao longo de uma geratriz (em pontos regulares) é constante.

Podemos distinguir dois casos (sem exaurir as possibilidades) de superfícies desenvolvíveis.

1. Se $\omega(t) \wedge \omega^{\prime}(t) \equiv 0$ então $\omega^{\prime}(t) \equiv 0$. Assim $w(t)$ é constante e a superfície é um cilindro sobre a curva obtida interceptando o cilindro com um plano normal a $\omega(t)$.

2. Se $\omega(t) \wedge \omega^{\prime}(t) \neq 0$, para todo $t \in I$, então $\omega^{\prime}(t) \neq 0$. Logo a superfície é não cilíndrica e podemos aplicar nosso trabalho prévio. Assim, podemos determinar a curva 
de estrição e verificar que o parâmetro de distribuição se anula:

$$
\lambda=\frac{\left(\beta, \omega, \omega^{\prime}\right)}{\left\|\omega^{\prime}\right\|^{2}} \equiv 0 .
$$

Portanto a curva de estrição será o lugar geométrico dos pontos singulares da superfície desenvolvível. Se $\beta^{\prime}(t) \neq 0$ para todo $t \in I$, segue de 1.1 e o do fato que $<\beta^{\prime}(t), \omega^{\prime}(t)>\equiv 0$ que $\omega$ é paralelo a $\beta^{\prime}$.

Logo a superfície regrada é a superfície tangente a $\beta$. Se $\beta^{\prime}(t)=0, \forall t \in I$, então a curva de estrição é um ponto, e a superfície regular é um cone com vértice em um ponto.

Esses não são os únicos casos de superfícies desenvolvíveis, novamente, se existe um ponto de acumulação dos zeros das funções envolvidas a análise deve ser mais cautelosa.

Exemplo 1.3.2 A envoltória da família dos planos tangentes ao longo de uma curva da superfície desenvolvível.

Seja $S$ uma superfície regular e $\alpha(s)$ uma curva em $S$ parametrizada por comprimento de arco. Assuma que $\alpha$ não é tangente a uma direção assintótica, isto é, $\alpha^{\prime}(s)$ não é uma direção assintótica portanto $k_{n}(s)=\Pi\left(\alpha^{\prime}(s)\right)=<d N\left(\alpha^{\prime}(s)\right), \alpha^{\prime}(s)>\neq 0$ assim $d N\left(\alpha^{\prime}(s)\right)=N^{\prime}(s) \neq 0$. Consideremos agora

$$
\chi(s, v)=\alpha(s)+v \frac{N(s) \wedge N^{\prime}(s)}{\left\|N^{\prime}(s)\right\|},
$$

onde $N(s)$ é o vetor unitário normal a $S$ restrito a curva $\alpha(s)$.

Mostremos que $\chi$ é uma superfície desenvolvível, de fato:

$$
\left\langle\frac{N \wedge N^{\prime}}{\left\|N^{\prime}\right\|} \wedge\left(\frac{N \wedge N^{\prime}}{\left\|N^{\prime}\right\|}\right)^{\prime}, \alpha^{\prime}\right\rangle=\frac{1}{\left\|N^{\prime}\right\|^{2}}\left\langle<N \wedge N^{\prime}, N^{\prime \prime}>N, \alpha^{\prime}\right\rangle=0 .
$$

Observemos também que $\chi$ é regular numa vizinhança de $v=0$ e é tangente a $S$ ao longo de $v=0$. Para provar isso temos que se $v=0$,

$$
\chi_{s} \wedge \chi_{v}=\alpha^{\prime} \wedge\left(\frac{N \wedge N^{\prime}}{\left\|N^{\prime}\right\|}\right)=<N^{\prime}, \alpha^{\prime}>\frac{N}{\left\|N^{\prime}\right\|}=-<N, \alpha^{\prime \prime}>\frac{N}{\left\|N^{\prime}\right\|}=-k_{n} \frac{N}{\left\|N^{\prime}\right\|},
$$

onde $k_{n}$ é a curvatura normal de $\alpha$. Portanto como $k_{n}$ é não nula temos que $\chi$ é regular numa vizinhança de $v=0$ e como

$$
\chi_{s} \wedge \chi_{v}=-k_{n} \frac{N}{\left\|N^{\prime}\right\|}
$$

temos que o vetor normal unitário de $\chi$ em $\chi(s, 0)$ é paralelo a $N(s)$.

Daremos agora uma interpretação geométrica para $\chi$. Consideremos a família $\left\{T_{\alpha(s)}(S)\right\}$ de planos tangentes a $S$ ao longo de $\alpha(s)$. 
Se $\Delta s$ é pequeno, dois planos $T_{\alpha(s)}(S)$ e $T_{\alpha(s)+\Delta s}(S)$ da família se interceptam ao longo de uma reta paralela ao vetor

$$
\frac{N(s) \wedge N(s+\Delta s)}{\Delta s} .
$$

Se $\Delta s \rightarrow 0$ esta reta aproxima-se da posição limite paralela ao vetor

$$
\lim _{\Delta s \rightarrow 0} \frac{N(s) \wedge(s+\Delta s)}{\Delta s}=\lim _{\Delta s \rightarrow 0} N(s) \wedge \frac{(N(s+\Delta s)-N(s))}{\Delta s}=N(s) \wedge N^{\prime}(s) .
$$

Intuitivamente isto significa que as geratrizes de $\chi$ são posições limites de intersecção de planos vizinhos da família $\left\{T_{\alpha(s)}(S)\right\}$, $\chi$ é chamada de envelope da família de planos tangentes da $S$ ao longo de $\alpha$.

Como sub-exemplos temos que se $\alpha$ é uma parametrização de um paralelo da esfera $S^{2}$ então o envelope dos planos tangentes de $S^{2}$, ao longo de $\alpha$, é um cilindro se o paralelo é um equador ou um cone, caso contrário. 


\section{Capítulo 2}

\section{Teoria de Singularidades}

Neste capítulo apresentamos as definições e principais resultados da teoria de singularidades que serão usados para o desenvolvimento do estudo das singularidades das superfícies regradas em $\mathbb{R}^{3}$. Estamos assumindo que o leitor já tenha algum conhecimento prévio nos deixando livres para omitir fatos elementares. As principais referências que utilizamos aqui são [7, 9, 15].

\subsection{Germes e Jatos}

Definição 2.1.1 Duas aplicações diferenciáveis $f_{i}: U_{i} \subset N \rightarrow P, i=1,2$, são equivalentes em $x \in N$ se existir uma vizinhança $U \subset U_{1} \cap U_{2}$ de $x \in N$ tal que $f_{1} \backslash_{U} \equiv f_{2} \backslash_{U}$. A classe de equivalência desta relação é chamada de germe de aplicação em $x$, um elemento da classe é chamado representante do germe, o elemento $x$ é chamado fonte e y de meta. Notação: $f:(N, x) \rightarrow(P, y)$ para $f(x)=y$.

Definição 2.1.2 Para cada germe $f:(N, x) \rightarrow(P, y)$ definimos a derivada $d_{x} f: T_{x} N \rightarrow$ $T_{y} P$ como sendo a derivada em $x$ de qualquer representante.

Definição 2.1.3 Dizemos que um germe $f:(N, x) \rightarrow(P, y)$ é um germe de difeomorfismo se, e somente se, um de seus representantes é um difeomorfismo.

Definição 2.1.4 O posto (co-posto) de um germe é definido como o posto (co-posto) de sua derivada em $x$. Quando o posto é igual a dimensão de $N$ o germe é imersivel e quando o posto é igual a dimensão de $P$ dizemos submersivel. Quando o germe não é imersivel nem submersivel em $x$ dizemos que $x$ é ponto singular. 
Definição 2.1.5 O espaço dos jatos $J^{k}(n, p)$ é o espaço vetorial real das aplicações $f: \mathbb{R}^{n} \rightarrow \mathbb{R}^{p}$ onde cada componente $f_{i}$ de $f$ é um polinômio de grau menor ou igual a $k$ nas coordenadas canônicas $x_{1}, \ldots, x_{n}$ de $\mathbb{R}^{n}$ com termo constante nulo. Os elementos de $J^{k}(n, p)$ são chamados de $k$-jatos.

Definição 2.1.6 Para cada $f \in C^{\infty}\left(\mathbb{R}^{n}, \mathbb{R}^{p}\right)$ e cada $a \in \mathbb{R}^{n}$, definimos a aplicação $j^{k} f: \mathbb{R}^{n} \rightarrow J^{k}(n, p)$ por $j^{k} f(a)$ como sendo o polinômio de Taylor de $f(x+a)-f(a)$ de ordem $k$ na origem.

\subsection{A topologia $C^{\infty}(X, Y)$ de Whitney}

Definição 2.2.1 Sejam $X$ e $Y$ variedades diferenciáveis. Fixemos um inteiro $k$ não negativo. Seja $U$ um subconjunto aberto de $J^{k}(X, Y)$. Então denotamos por $M(U)$ o conjunto $\left\{f \in C^{\infty}(X, Y) / j^{k} f(X) \subset U\right\}$. A família de conjuntos $\left\{M(U) / U \subset J^{k}(X, Y)\right\}$ forma uma base para a topologia $C^{k}$ de Whitney (ou $C^{k}$-topologia).

Definição 2.2.2 Sejam $X$ e $Y$ variedades diferenciáveis. Denotemos por $W_{k}$ o conjunto dos subconjuntos abertos de $C^{\infty}(X, Y)$ na topologia $C^{k}$. A topologia $C^{\infty}$ de Whitney sobre $C^{\infty}(X, Y)$ é a topologia cuja base é $W=\bigcup_{k=0}^{\infty} W_{k}$.

A base acima está bem definida pois $W_{k} \subset W_{l}$ sempre que $k \leq l$. Para ver isto basta usar a projeção canônica $\pi_{k}^{l}: J^{l}(X, Y) \rightarrow J^{k}(X, Y)$ onde $\pi_{k}^{l}(\sigma)=j^{k} \sigma$. Então $M(U)=M\left(\left(\pi_{k}^{l}\right)^{-1}(U)\right)$ para todo conjunto aberto $U$ em $J^{k}(X, Y)$.

Não é difícil mostrar que uma vizinhança básica na topologia $C^{k}$ de Whitney pode ser dada por $B_{\rho}(f)=\left\{g \in C^{\infty}(X, Y) / \forall x \in X, d\left(j^{k} f(x), j^{k} g(x)\right)<\rho(x)\right\}$ onde $d$ é uma métrica em $J^{k}(X, Y)$ e $\rho: X \rightarrow \mathbb{R}^{+}$é contínua. Podemos pensar em $B_{\rho}(f)$ como o conjunto da aplicações diferenciáveis de $\mathrm{X}$ em $\mathrm{Y}$ que têm as primeiras $k$ derivadas $\rho$ próximas de $f$.

Definição 2.2.3 Seja $X$ um espaço topológico. Então:

i) Um subconjunto $G \in X$ é residual se ele é a intersecção enumerável de subconjuntos abertos e densos de $X$.

ii) X é um espaço de Baire se todo conjunto residual é denso.

As demonstrações dos resultados abaixo podem ser encontradas em [9].

Proposição 2.2.4 Sejam $X$ e $Y$ variedades diferenciáveis. Então $C^{\infty}(X, Y)$ é um espaço de Baire na topologia $C^{\infty}$ de Whitney. 
Proposição 2.2.5 Sejam $X$ e $Y$ variedades diferenciáveis. Então $j^{k}: C^{\infty}(X, Y) \rightarrow$ $C^{\infty}\left(X, J^{k}(X, Y)\right)$ definida por $f \mapsto j^{k} f$ é contínua na topologia $C^{\infty}$ de Whitney.

Proposição 2.2.6 Sejam $X, Y$ e $Z$ variedades diferenciáveis. Então $C^{\infty}(X, Y) \times C^{\infty}(X, Z)$ é homeomorfo, na topologia $C^{\infty}$ de Whitney, a $C^{\infty}(X, Y \times Z)$.

\subsection{Ação de Grupos de Lie em Variedades}

Definição 2.3.1 Uma ação de um grupo $G$ em um conjunto $M$ é uma aplicação $\varphi$ : $G \times M \rightarrow M$, denotada por $\varphi(g, x)=g x$ tal que para todo $x \in M$ e $g, h \in G$ temos:

i) $1 x=x$, com 1 identidade de $G$;

ii) $(g h) x=g(h x)$.

O conjunto $G x=\{g x \in M / g \in G\}$ é chamado órbita de $x$ em $M$. A relação dada por $y \sim x$ se $y \in G x$ é uma relação de equivalência.

Definição 2.3.2 Um grupo de Lie é um grupo multiplicativo G que é uma variedade diferenciável onde as operações de multiplicação e inversão são diferenciáveis. Uma ação de grupo de Lie é uma ação de um grupo de Lie G em uma variedade diferenciável $M$ que seja diferenciável.

Em geral, as órbitas de uma ação de grupo de Lie numa variedade não são subvariedades e sim subvariedades imersas, mas nos casos em que $G x$ é variedade, vale o seguinte resultado:

Teorema 2.3.3 Seja $\varphi: G \times M \rightarrow M$ uma ação de grupo de Lie $G$ em uma variedade $M$. Se as órbitas são subvariedades de $M$, então para todo $x \in M$ a aplicação $\varphi_{x}: G \rightarrow G_{x}$ dada por $\varphi_{x}(g)=g x$ é uma submersão. E mais, o espaço tangente à órbita Gx em $x$ é a imagem de $d_{1} \varphi_{x}: T_{1} G \rightarrow T_{x} M$, isto é, $T_{x} G x=d_{1} \varphi_{x}\left(T_{1} G\right)$.

Demonstração: Ver [7].

\subsection{Transversalidade}

Definição 2.4.1 Dizemos que duas subvariedades $N_{1}, N_{2} \subset N$ de uma variedade diferenciável $N$ se interceptam transversalmente em $x \in N_{1} \cap N_{2}$ quando $T_{x} N_{1}+T_{x} N_{2}=T_{x} N$. Quando $N_{1}$ e $N_{2}$ se interceptam transversalmente em todo $x \in N_{1} \cap N_{2}$ dizemos que são transversais. 
Definição 2.4.2 Seja $f: N \rightarrow P$ uma aplicação diferenciável entre variedades. Seja $Q \subset P$ uma subvariedade. Dizemos que $f$ é transversal a $Q(f \pitchfork Q)$ quando:

i) $f(x) \notin Q$, ou

ii) $\forall x \in N$ com $y=f(x) \in Q$ tem-se que

$$
d_{x} f\left(T_{x} N\right)+T_{y} Q=T_{y} P
$$

Proposição 2.4.3 Sejam $f: N \rightarrow P$ uma aplicação diferenciável entre variedades e $Q \subset$ $P$ uma subvariedade com $f \pitchfork Q$. Então $M=f^{-1}(Q)$ é subvariedade de $N$ tendo a mesma codimensão que $Q$ ou é vazio. Além disso, para todo ponto $x \in M \operatorname{com} y=f(x) \in Q$ temos $T_{x} M=d_{x} f^{-1}\left(T_{y} Q\right)$.

Demonstração: Ver [7].

Proposição 2.4.4 Sejam $Q_{1}, \ldots, Q_{t} \subset \mathbb{R}^{p}$ subvariedades então o conjunto das aplicações diferenciáveis de $\mathbb{R}^{n}$ em $\mathbb{R}^{p}$ transversais a $Q_{i}, i=1, \ldots, t$, é denso em $C^{\infty}\left(\mathbb{R}^{n}, \mathbb{R}^{p}\right)$.

Demonstração: Ver [7].

Proposição 2.4.5 (Teorema de Transversalidade de Thom) Sejam $Q_{1}, \ldots, Q_{t}$ subvariedades diferenciáveis do espaço de jatos $J^{k}(n, p)$. O conjunto de todas as aplicações $f$ : $\mathbb{R}^{n} \rightarrow \mathbb{R}^{p}$ para as quais $j^{k} f: \mathbb{R}^{n} \rightarrow J^{k}(n, p)$ é transversal a $Q_{1}, \ldots, Q_{t}$ é denso em $C^{\infty}\left(\mathbb{R}^{n}, \mathbb{R}^{p}\right)$.

Demonstração: Ver [7].

\subsection{Genericidade e Estabilidade}

Definição 2.5.1 Seja $S$ um espaço topológico. Uma propriedade $P$ de elementos de $S$ é genérica se o conjunto de todos os $x \in S$ satisfazendo $P$ contém um conjunto $A$ o qual deve ser uma intersecção enumerável de conjuntos abertos e densos.

Observe que $A$ é um conjunto residual, assim quando $S$ é um espaço de Baire temos que $A$ é um conjunto denso de $S$ e dessa forma a propriedade $P$ é válida para um conjunto denso de $S$.

Definição 2.5.2 Seja $S$ um conjunto com uma topologia e uma relação de equivalência $\sim$. Um elemento $x \in S$ é estável (relativamente $a \sim$ ) se a classe de equivalência de $x$ contém uma vizinhança de $x$. 


\subsubsection{Estabilidade}

Definição 2.5.3 Sejam $f, g \in C^{\infty}(X, Y)$. Então $f \sim g(f$ é equivalente a $g$, ou $\mathcal{A}$ equivalente) se existem difeomorfismos $h: X \rightarrow X$ e $k: Y \rightarrow Y$ tais que o seguinte diagrama

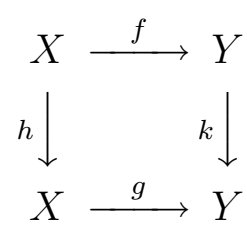

comuta. Sob esta relação de equivalência dizemos que $f$ é estável se existe uma vizinhança $W_{f}$ de $f$ em $C^{\infty}(X, Y)$ tal que se $g \in W_{f}$ então $g \sim f$.

A relação definida acima é uma relação de equivalência que pode ser vista como a ação do grupo $\mathcal{A}=\operatorname{Diff}(X) \times \operatorname{Diff}(Y)$ no conjunto $C^{\infty}(X, Y)$ dada por $\varphi((h, x), f)=k \circ f \circ h^{-1}$. Assim definida, as classes de equivalência são dadas pelas órbitas da ação.

Lema 2.5.4 Seja $f \in C^{\infty}(X, Y)$ então $f$ é estável se, e somente se, a órbita de $f$ em $C^{\infty}(X, Y)$ sob a ação do grupo $\mathcal{A}$ é um subconjunto aberto.

Demonstração: Ver [9].

Esta definição de estabilidade é difícil de ser verificada na prática. Na década de 60 John Mather [17] introduziu o conceito de estabilidade infinitesimal, o que trouxe um método computável para determinar se uma aplicação é ou não estável.

Definição 2.5.5 Seja $f \in C^{\infty}(X, Y)$.

a) Sejam $\pi_{Y}: T Y \rightarrow Y$ a projeção canônica do fibrado tangente $T Y=\bigcup_{y \in Y} T_{y} Y$ em $Y$ e $w: X \rightarrow T Y$ diferenciável. Então w é um campo de vetor ao longo de $f$ se o seguinte diagrama

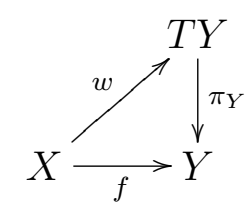

comuta. O conjunto dos campos de vetores ao longo de $f$ é denotado por $C_{f}^{\infty}(X, T Y)$.

b) $f$ é infinitesimalmente estável se para todo $w$ campo de vetores ao longo de $f$, existir um campo de vetores s sobre $X$ e um campo de vetores $t$ sobre $Y$ tais que

$$
w=(d f) . s+t . f
$$

onde $d f: T X \rightarrow T Y$ é tal que $d f(x, \nu(x))=d f_{x}(\nu(x))$ onde $d f_{x}$ é a matriz jacobiana de $f$ em $x$. 
Teorema 2.5.6 (Mather) Seja $X$ uma variedade compacta e $f: X \rightarrow Y$ diferenciável. Então f é estável se, e somente se, é infinitesimalmente estável.

Demonstração: Ver [9].

A caracterização das aplicações estáveis depende de maneira bastante importante do estudo do comportamento local das singularidades. Nas próximas seções veremos os principais conceitos e resultados que permitem efetuar esta classificação para o caso particular de aplicações de superfícies em $\mathbb{R}^{3}$.

\subsection{Conjunto de Singularidades de $1^{\text {a }}$ Ordem}

Definição 2.6.1 Seja $f: N \rightarrow P$, definimos por conjunto singular de primeira ordem o conjunto $\Sigma^{i} f=\left\{x \in N / \operatorname{dim} \operatorname{Ker} d_{x} f=i\right\}$. Definimos também o conjunto $\Sigma^{i}=\{\varphi \in$ $\left.J^{k}(n, p) / \operatorname{dim} \operatorname{Ker} \varphi=i\right\}$.

Lembremos que o posto de $f$ é menor ou igual a $\min \{n, p\}$ assim os conjuntos singulares particionam a variedade $N$ em um número finito de conjuntos nos quais $f$ tem posto constante. Seria útil se estes conjuntos fossem subvariedades, mas isso não é sempre verdade.

Teorema 2.6.2 $\Sigma^{i}$ é uma subvariedade diferenciável de $J^{1}(n, p)$ de codimensão $i(p-n+i)$.

Demonstração: Ver [7].

Proposição 2.6.3 Existe um conjunto denso de aplicações $f: \mathbb{R}^{n} \rightarrow \mathbb{R}^{p}$ para o qual $j^{1} f$ é transversal a todos os conjuntos $\Sigma^{i}$ e portanto para o qual cada $\Sigma^{i} f$ é uma variedade diferenciável de codimensão $i(p-n+i)$.

Demonstração: Segue facilmente do teorema de Transversalidade de Thom (Teorema $2.4 .5)$.

\section{$2.7 \mathcal{A}$-equivalência e $\mathcal{K}$-equivalência}

Definição 2.7.1 Denotamos por $\varepsilon_{n, p}$ o conjunto dos germes de aplicações $f:\left(\mathbb{R}^{n}, 0\right) \rightarrow$ $\mathbb{R}^{p}$ de classe $C^{\infty}$. Quando $p=1$ denotamos por $\varepsilon_{n}$. Denotamos $\varepsilon_{n, p}^{0}$ o conjunto dos germes $f:\left(\mathbb{R}^{n}, 0\right) \rightarrow\left(\mathbb{R}^{p}, 0\right)$. 
Proposição 2.7.2 $\varepsilon_{n}$ é um anel local cujo ideal maximal é $m_{n}=\left\{f \in \varepsilon_{n} / f(0)=0\right\}$. $\varepsilon_{n, p}$ é um $\varepsilon_{n}$-módulo livre de posto $p$.

Definição 2.7.3 Dois germes $f_{i}:\left(N_{i}, x_{i}\right) \rightarrow\left(P_{i}, y_{i}\right), i=1,2$, são $\mathcal{A}$-equivalentes se existem germes de difeomorfismos $h:\left(N_{1}, x_{1}\right) \rightarrow\left(N_{2}, x_{2}\right)$ e $k:\left(P_{1}, y_{1}\right) \rightarrow\left(P_{2}, y_{2}\right)$ tais que o seguinte diagrama

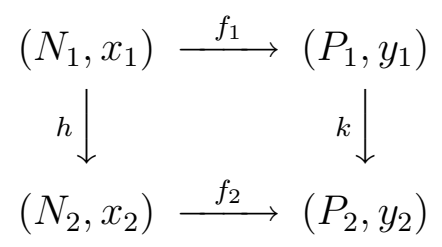

comuta.

Como qualquer germe $f:(N, x) \rightarrow(P, y)$ é $\mathcal{A}$-equivalente a algum germe de $\left(\mathbb{R}^{n}, 0\right)$ em $\left(\mathbb{R}^{p}, 0\right)$, então consideremos apenas germes de $\left(\mathbb{R}^{n}, 0\right) \rightarrow\left(\mathbb{R}^{p}, 0\right)$.

Definição 2.7.4 Dizemos que dois germes $f_{i}:\left(N_{i}, x_{i}\right) \rightarrow\left(P_{i}, y_{i}\right), i=1,2$ são $\mathcal{K}$ equivalentes quando existe $(h, H)$ um par de germes invertíveis, chamado equivalência de contato (ou $\mathcal{K}$-equivalência), satisfazendo o seguinte diagrama comutativo

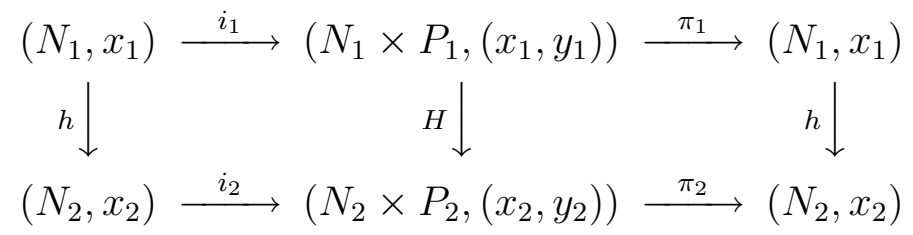

com $i_{k}$ inclusões e $\pi_{k}$ projeções, para os quais $H \circ\left(1, f_{1}\right)=\left(1, f_{2}\right) \circ h$ onde 1 é o germe identidade.

Temos assim $H(x, y)=(h(x), \theta(x, y)) \operatorname{com} \theta\left(x, y_{1}\right)=y_{2}$.

De maneira análoga a que foi feita para a $\mathcal{A}$-equivalência consideraremos, quando conveniente, apenas germes de $\left(\mathbb{R}^{n}, 0\right) \rightarrow\left(\mathbb{R}^{p}, 0\right)$.

Proposição 2.7.5 O conjunto $\mathcal{K}_{n, p}$ de todos os germes $H$ para os quais existe $(h, H)$ $\mathcal{K}$-equivalências com $h:\left(\mathbb{R}^{n}, 0\right) \rightarrow\left(\mathbb{R}^{n}, 0\right)$ e $H:\left(\mathbb{R}^{n} \times \mathbb{R}^{p}, 0\right) \rightarrow\left(\mathbb{R}^{n} \times \mathbb{R}^{p}, 0\right)$ é um grupo com a operação composição.

Proposição 2.7.6 Consideremos o grupo $\mathcal{K}_{n, p}$ e o conjunto $\varepsilon_{n, p}^{0}$ então a aplicação dada por $(h, H) . f=H \circ(1, f) \circ h^{-1}$ é uma ação. E mais, dois germes são $\mathcal{K}$-equivalentes se, e somente se, estão na mesma órbita.

Definição 2.7.7 Dado $f:\left(\mathbb{R}^{n}, 0\right) \rightarrow\left(\mathbb{R}^{p}, 0\right)$ definimos o pull back de $f$, por $f^{*}: \varepsilon_{p, t} \rightarrow$ $\varepsilon_{n, t}$ onde $f^{*}(h)=h \circ f$. 
Teorema 2.7.8 Uma condição necessária e suficiente para que $f, g:\left(\mathbb{R}^{n}, 0\right) \rightarrow\left(\mathbb{R}^{p}, 0\right)$ sejam $\mathcal{K}$-equivalentes é que exista $\phi:\left(\mathbb{R}^{n}, 0\right) \rightarrow\left(\mathbb{R}^{n}, 0\right)$ germe de difeomorfismo tal que $\phi^{*}\left(g^{*}\left(m_{p}\right) \varepsilon_{n}\right)=f^{*}\left(m_{p}\right) \varepsilon_{n}$, onde $\phi^{*}, g^{*}$ e $f^{*}$ são o pull back de $\phi, g$ e $f$ respectivamente.

Podemos dar agora a versão local de estabilidade infinitesimal.

Definição 2.7.9 Dada $f:\left(\mathbb{R}^{n}, 0\right) \rightarrow\left(\mathbb{R}^{p}, 0\right)$ dizemos que $f$ é infinitesimalmente estável se

$$
\varepsilon_{n, p}=\left\langle\frac{\partial f}{\partial x_{1}}, \ldots, \frac{\partial f}{\partial x_{n}}\right\rangle_{\varepsilon_{n}}+f^{*} \varepsilon_{p, p}
$$

onde $f^{*}(\eta)=\eta \circ f$, para $\eta \in \varepsilon_{p, p}$.

Teorema 2.7.10 O guarda-chuva de Whitney, dado por $(x, y) \mapsto\left(x^{2}, y, x y\right)$, é infinitesimalmente estável.

A demonstração deste teorema será dada na próxima seção.

\subsection{Determinação Finita e Transversal Completa}

Definição 2.8.1 Definimos o grupo $\mathcal{A}_{1}$ como sendo o subgrupo de $\mathcal{A}$ dos elementos que tem o 1-jato igual a identidade.

Definição 2.8.2 Um germe $f \in \varepsilon_{n, p}^{0}$ é $k$-A-determinado se todo germe $g \in \varepsilon_{n, p}^{0}$ com mesmo k-jato que $f$ é $\mathcal{A}$-equivalente a $f$. Dizemos que $f$ é finitamente $\mathcal{A}$-determinado se é $k$-A-determinado para algum $k$. A mesma terminologia é usada para o grupo $\mathcal{K}$ e $\mathcal{A}_{1}$.

A investigação da determinação finita começou com os trabalhos de John Mather em 1960, [16, 17, 18]. Mather deu uma primeira estimativa do grau de determinação finita de um germe de aplicação. Resultados de T. Gaffney [6] e A. du Plessis [23], nas décadas seguintes, melhoraram bastante estas estimativas. Em 1987 o artigo [2] resolveu o problema completamente. Os resultados centrais de determinação decorrentes de todos estes trabalhos estão contidos no Teorema 2.8.3.

Indiquemos por $T \mathcal{A} f$ e $T \mathcal{A}_{1} f$ os espaços tangentes aos grupos $\mathcal{A}$ e $\mathcal{A}_{1}$ respectivamente e por $T \mathcal{A}_{e} f$ o espaço tangente estendido. Para $f: \mathbb{R}^{2} \rightarrow \mathbb{R}^{3}$, estes espaços são dados por:

$$
T_{f} \mathcal{A} f=\left\langle\frac{\partial f}{\partial x}, \frac{\partial f}{\partial y}\right\rangle_{m_{2}}+f^{*}\left(m_{3} \varepsilon_{3,3}\right)
$$




$$
\begin{gathered}
T_{f} \mathcal{A}_{1} f=\left\langle\frac{\partial f}{\partial x}, \frac{\partial f}{\partial y}\right\rangle_{m_{2}^{2}}+f^{*}\left(m_{3}^{2} \varepsilon_{3,3}\right) \\
T_{f} \mathcal{A}_{e} f=\left\langle\frac{\partial f}{\partial x}, \frac{\partial f}{\partial y}\right\rangle_{\varepsilon_{2}}+f^{*} \varepsilon_{3,3}
\end{gathered}
$$

Teorema 2.8.3 (1) Um germe $f \in \varepsilon_{n, p}^{0}$ é finitamente $\mathcal{A}$-determinado se, e somente se, existe um inteiro positivo $k$, tal que $m_{n}^{k} \cdot \varepsilon_{n, p} \subset T \mathcal{A} f$.

(2) Um germe $f \in \varepsilon_{n, p}^{0}$ é (2r+1)-A-determinado se tivermos $m_{n}^{r+1} \cdot \varepsilon_{n, p} \subset T \mathcal{A} f+$ $m_{n}^{2 r+2} \cdot \varepsilon_{n, p}$.

(3) Um germe $f \in \varepsilon_{n, p}^{0}$ é $r$ - $\mathcal{A}_{1}$-determinado se, e somente se, tivermos $m_{n}^{r+1} \varepsilon_{n, p} \subset$ $T \mathcal{A}_{1} f$.

(4) Um germe $f \in \varepsilon_{n, p}^{0}$ é $r$ - $\mathcal{A}_{1}$-determinado se, e somente se, tivermos $m_{n}^{r+1} \cdot \varepsilon_{n, p} \subset$ $T \mathcal{A}_{1} f+m_{n}^{r+1} \cdot\left(f^{*} m_{p} \cdot \varepsilon_{n}+m_{n}^{r+1}\right) \cdot \varepsilon_{n, p}$.

Colorário 2.8.4 Um germe $f \in \varepsilon_{n, p}^{0}$ é $r$ - $\mathcal{A}_{1}$-determinado se, e somente se, tivermos

$$
m_{n}^{r+1} \cdot \varepsilon_{n, p} \subset T \mathcal{A}_{1} f+m_{n}^{2 r+2} \cdot \varepsilon_{n, p} .
$$

Proposição 2.8.5 (Transversal Completa para Jatos) Seja $f \in m_{n} \varepsilon_{n, p}$ e $T \subset H^{k+1}(n, p)$ tais que

$$
H^{k+1}(n, p) \subset T\left(J^{k+1} \mathcal{A}_{1}\right) j^{k+1} f+T
$$

então todo $(k+1)$-jato $j^{k} g$, com $j^{k} g=j^{k} f$, está na mesma $\mathcal{A}_{1}$-órbita de $j^{k+1} f+t$ para algum $t \in T$.

Demonstração: Ver [5].

Proposição 2.8.6 O guarda-chuva de Whitney é 2 - $\mathcal{A}_{1}$-determinado.

Demonstração: Consideremos o guarda-chuva de Whitney $f: \mathbb{R}^{2} \rightarrow \mathbb{R}^{3}$ definido por $f(x, y)=\left(x^{2}, y, x y\right)$. Inicialmente temos que $\mathcal{A}_{1}$ é sub-grupo de $\mathcal{A}$ cujos elementos tem o 1-jato igual a identidade. Assim

$$
T_{f} \mathcal{A}_{1} f=\left\langle\frac{\partial f}{\partial x}, \frac{\partial f}{\partial y}\right\rangle_{m_{2}^{2}}+f^{*}\left(m_{2}^{3} \varepsilon_{3,3}\right)=\left\langle\left(\begin{array}{c}
2 x \\
0 \\
y
\end{array}\right),\left(\begin{array}{c}
0 \\
1 \\
x
\end{array}\right)\right\rangle_{m_{2}^{2}}+\left(\begin{array}{c}
\eta_{1}\left(x^{2}, y, x y\right) \\
\eta_{2}\left(x^{2}, y, x y\right) \\
\eta_{3}\left(x^{2}, y, x y\right)
\end{array}\right)
$$

$\operatorname{com} \eta_{i} \in m_{3}^{2}$ 
Calcular a 2-transversal completa de $f$ significa encontrar um espaço vetorial $T$ tal que $H^{3}(2,3) \subset\left(T_{f} \mathcal{A}_{1} f+T\right) \bmod m_{2}^{4}$. Deste modo temos que $m_{2}^{3} \varepsilon_{2,3} \subset T_{f} \mathcal{A}_{1} f+T+m_{2}^{4} \varepsilon_{2,3}$. Assim se $T=\emptyset$ teremos $m_{2}^{3} \varepsilon_{2,3} \subset T_{f} \mathcal{A}_{1} f+m_{2}^{4} \varepsilon_{2,3}$.

Pelo corolário anterior se a $i$-transversal completa de $f$ for vazia para $2 \leq i \leq 5$, então $f$ é 2-determinada.

Vejamos quais elementos de $H^{3}(2,3)$ estão em $T_{f} \mathcal{A}_{1} f$. Tomando apenas $\eta_{i}\left(x^{2}, y, x y\right)=$ $y^{3}, \eta_{j}\left(x^{2}, y, x y\right)=x^{2} y$ e $\eta_{k}\left(x^{2}, y, x y\right)=x y^{2}$, para $i, j$ e $k$ convenientes, podemos gerar os seguintes elementos:

$$
\left(\begin{array}{c}
y^{3} \\
0 \\
0
\end{array}\right), \ldots,\left(\begin{array}{c}
0 \\
0 \\
y^{3}
\end{array}\right),\left(\begin{array}{c}
x^{2} y \\
0 \\
0
\end{array}\right), \ldots,\left(\begin{array}{c}
0 \\
0 \\
x^{2} y
\end{array}\right),\left(\begin{array}{c}
x y^{2} \\
0 \\
0
\end{array}\right),\left(\begin{array}{c}
0 \\
x y^{2} \\
0
\end{array}\right) \mathrm{e}\left(\begin{array}{c}
0 \\
0 \\
x y^{2}
\end{array}\right) .
$$

Finalmente

$$
\left(\begin{array}{c}
x^{3} \\
0 \\
0
\end{array}\right)=\frac{1}{2}\left(\begin{array}{cc}
2 x & 0 \\
0 & 1 \\
y & x
\end{array}\right) \cdot\left(\begin{array}{c}
x^{2} \\
0
\end{array}\right)-\left(\begin{array}{c}
0 \\
0 \\
x^{2} y
\end{array}\right)
$$

Analogamente podemos mostrar que $\left(0, x^{3}, 0\right)$ e $\left(0,0, x^{3}\right)$ também estão em $T_{f} \mathcal{A}_{1} f$, assim $H^{3}(2,3) \subset\left(T_{f} \mathcal{A}_{1} f\right) \bmod m_{2}^{4} \operatorname{logo}$ a 2 -transversal completa de $f$ é vazia.

O mesmo método nos leva a concluir que a $i$-transversal completa de $f$ é vazia para $3 \leq i \leq 5$ e disto segue o resultado.

Podemos agora demonstrar o Teorema 2.7.10.

Demonstração: (Demonstração do Teorema 2.7.10) Para provar que o guarda-chuva de Whitney é infinitesimalmente estável, basta mostrar que o espaço tangente estendido de $f$ (veja definição 2.7.9) satisfaz à condição $T \mathcal{A}_{e} f=\varepsilon_{n, p}$.

É claro que $T \mathcal{A}_{e} f \supset T \mathcal{A} f \supset T \mathcal{A}_{1} f$. Assim, dos cálculos realizados para a proposição 2.8.6, segue-se que $T \mathcal{A}_{e} f \supset m_{3}^{2} \varepsilon_{2,3}$. Não é difícil agora verificar que $T \mathcal{A}_{e} f$ contém todas as aplicações polinomiais de grau menor ou igual a 2 e portanto $T \mathcal{A}_{e} f=\varepsilon_{2,3}$. 


\section{Capítulo 3}

\section{Aplicações de $M^{2}$ em $\mathbb{R}^{3}$}

Neste capítulo estaremos interessados em caracterizar as aplicações estáveis de $M^{2}$ em $\mathbb{R}^{3}$, e mostrar que as aplicações estáveis nestas dimensões formam um conjunto denso. Estes resultados foram demonstrados por H. Whitney em 1944 (ver [27]).

Em nossa abordagem, procuramos utilizar resultados recentes da teoria, permitindo assim cálculos mais simples do que os originalmente efetuados pro Whitney. As demonstrações de alguns teoremas são apenas indicadas ou mesmo omitidas. Nestes casos, maiores detalhes podem ser obtidos em [9].

\subsection{Caracterização de Aplicações Estáveis}

Proposição 3.1.1 Seja $f: M^{2} \rightarrow \mathbb{R}^{3}$ aplicação estável. Então $j^{1} f \pitchfork \Sigma^{i}$ para todo $i$.

Demonstração: Sabemos que o conjunto das $f \in C^{\infty}\left(M^{2}, \mathbb{R}^{3}\right)$ tais que $j^{1} f \pitchfork \Sigma^{i}$, para todo $i$, é denso. Como $f$ é estável existe uma vizinhança $V$ de $f$ tal que todo elemento de $V$ é equivalente a $f$. Em particular existe $g \in V$ tal que $g \pitchfork \Sigma^{i}$ para todo $i$ e $g \sim f$ portanto $f \pitchfork \Sigma^{i}$ para todo $i$.

Observação 3.1.2 Segundo a definição 2.6.1 e o teorema 2.6.2 podemos fazer a seguinte observação. Seja $f: M^{2} \rightarrow \mathbb{R}^{3}$, tal que $j^{1} f \pitchfork \Sigma^{i}$, para todo $i$, então codim $\Sigma^{i}=i(i+1)$ logo:

i) se $i=0$ então codim $\Sigma^{0}=0$ portanto $\Sigma^{0}(f)$ tem dimensão 2 e é um aberto de $M$;

ii) se $i=1$ então codim $\Sigma^{1}=2$ portanto $\Sigma^{1}(f)$ tem dimensão 0;

iii) se $i \geq 2$ então codim $\Sigma^{i}>\operatorname{dim} M$ portanto $\Sigma^{i}(f)=\emptyset$. 
Colorário 3.1.3 Seja $f: M^{2} \rightarrow \mathbb{R}^{3}$ uma aplicação estável. Então:

a) $\operatorname{dim} \Sigma^{0}(f)=2$;

b) $\operatorname{dim} \Sigma^{1}(f)=0$;

c) $\Sigma^{2}(f)=\emptyset$.

Definição 3.1.4 Sejam $f: X \rightarrow Y$ diferenciável e $f^{(s)}: X^{(s)} \rightarrow Y^{s}$ a restrição de $f \times \ldots \times f: X \times \ldots \times X \rightarrow Y^{s}$ ao conjunto $X^{(s)}=\left\{\left(x_{1}, \ldots, x_{s}\right) \in X^{s} / x_{i} \neq x_{j}, j \neq i\right\}$. Consideremos a diagonal $\Delta Y^{s}=\left\{(y, \ldots, y) \in Y^{s} / y \in Y\right\}$. Então $f$ é uma aplicação com cruzamento normal se $f^{(s)} \pitchfork \Delta Y^{s}$, para todo $s>1$.

Proposição 3.1.5 Seja $X$ compacto (ou $f$ própria). Então o conjunto das aplicações de $X$ em $Y$ com cruzamento normal é denso em $C^{\infty}(X, Y)$. (ou em $C_{p r}^{\infty}(X, Y)$, conjunto das aplicações próprias de $X$ em $Y$ ).

Demonstração: A demonstração segue do teorema de Transversalidade de Thom (Teorema 2.4 .5$)$.

Colorário 3.1.6 O conjunto das imersões com cruzamento normal é denso no conjunto das imersões.

Teorema 3.1.7 Seja $f \in C^{\infty}(X, Y)$ uma imersão. Então $f$ é estável se, e somente se, $f$ tem somente cruzamentos normais.

Demonstração: Como o conjunto das imersões com cruzamentos normais é denso no conjunto da imersões, dada $f: X \rightarrow Y$ imersão estável, existe $V_{f}$ uma vizinhança de $f$ tal que todo $g \in V_{f}$ é equivalente a $f$ por outro lado $V_{f}$ contém alguma $g$ que é uma imersão com cruzamento normal. Como $g$ é equivalente a $f, f$ também possui esta propriedade.

A recíproca consiste em mostrar que toda imersão com cruzamento normal é infinitesimalmente estável e portanto, estável.

Proposição 3.1.8 Seja $f: M^{2} \rightarrow \mathbb{R}^{3}$ tal que $f^{(s)}: M^{(s)} \rightarrow\left(\mathbb{R}^{3}\right)^{s}$ é transversal a $\Delta\left(\mathbb{R}^{3}\right)^{s}$ então $\left(f^{s}\right)^{-1}\left(\Delta\left(\mathbb{R}^{3}\right)^{s}\right)=\emptyset$ se $s \geq 4$.

Demonstração: É fácil notar que $\Delta\left(\mathbb{R}^{3}\right)^{s} \subset\left(\mathbb{R}^{3}\right)^{s}$ é subvariedade diferenciável e que $f^{(s)}: M^{(s)} \rightarrow\left(\mathbb{R}^{3}\right)^{s}$ é diferenciável. Como $f^{(s)} \pitchfork \Delta\left(\mathbb{R}^{3}\right)^{s}$ temos que $N=\left(f^{(s)}\right)^{-1}\left(\Delta\left(\mathbb{R}^{3}\right)^{s}\right)$ é subvariedade de mesma codimensão que $\Delta\left(\mathbb{R}^{3}\right)^{s}$ ou então $N$ é vazio. Portanto como codim $\Delta\left(\mathbb{R}^{3}\right)^{s}=3(s-1)$ temos que codim $N=3(s-1)$ e se $s \geq 4$ então codim $\mathrm{N}>$ $\operatorname{dim} M^{(s)}$ o que é absurdo, pois $N \subset M^{(s)}$ assim $N=\emptyset$ para $s \geq 4$. 
Teorema 3.1.9 Seja $f: M^{2} \rightarrow \mathbb{R}^{3}$ com $M$ compacto ou $f$ própria. Então temos que $j^{1} f \pitchfork \Sigma^{i}$ para todo $i$ se, e somente se, as únicas singularidades de $f$ são guarda-chuvas de Whitney.

Demonstração: Segue do corolário 3.1.3 que basta considerar $i=1$. Além disso, precisamos apenas fazer o estudo local, na vizinhança de um ponto singular de tipo $\Sigma^{1}$.

Se $f$ é equivalente ao guarda-chuva de Whitney, na vizinhança de um ponto $p \in$ $M$, o germe de $f$ no ponto singular $p$ é infinitesimalmente estável. Logo para qualquer representante de $f$, segue da proposição 3.1.1 que $j^{1} f \pitchfork \Sigma^{1}$ em $p$.

Para demonstrar a recíproca demonstraremos as seguintes afirmações:

1) Nas condições do teorema $f$ é equivalente a $\left(x, b_{1} x y+c_{1} y^{2}+R_{2}, b_{2} x y+c_{2} y^{2}+R_{3}\right)$.

2) Se $j^{1} f \pitchfork \Sigma^{i}$ então $b_{1} c_{2}-b_{2} c_{2} \neq 0$.

Para demonstrar 1) observemos que $f$ tem co-posto 1 logo existem transformações lineares $P \in G L(3)$ e $Q \in G L(2)$ tais que a expansão de Taylor de $P \circ f \circ Q$ tem a seguinte forma:

$$
\begin{aligned}
g(x, y)=P \circ f \circ & Q(x, y)=\left(x+\frac{1}{2} \frac{\partial^{2} f_{1}}{\partial x^{2}}(0,0) x^{2}+\frac{\partial^{2} f_{1}}{\partial x y}(0,0) x y+\frac{1}{2} \frac{\partial^{2} f_{1}}{\partial y^{2}}(0,0) y^{2}+R_{1},\right. \\
& \frac{1}{2} \frac{\partial^{2} f_{2}}{\partial x^{2}}(0,0) x^{2}+\frac{\partial^{2} f_{2}}{\partial x y}(0,0) x y+\frac{1}{2} \frac{\partial^{2} f_{2}}{\partial y^{2}}(0,0) y^{2}+R_{2}, \\
& \left.\frac{1}{2} \frac{\partial^{2} f_{3}}{\partial x^{2}}(0,0) x^{2}+\frac{\partial^{2} f_{3}}{\partial x y}(0,0) x y+\frac{1}{2} \frac{\partial^{2} f_{3}}{\partial y^{2}}(0,0) y^{2}+R_{3}\right) .
\end{aligned}
$$

Portanto $f$ é equivalente a $g(x, y)=\left(x+a_{1} x^{2}+b_{1} x y+c_{1} y^{2}+R_{1}, a_{2} x^{2}+b_{2} x y+c_{2} y^{2}+\right.$ $\left.R_{2}, a_{3} x^{2}+b_{3} x y+c_{3} y^{2}+R_{3}\right)$.

Efetuando a seguinte mudança de coordenadas

$$
\left\{\begin{array}{l}
X=x+a_{1} x^{2}+b_{1} x y+c_{1} y^{2}+R_{1} \\
Y=y \\
Z=z
\end{array}\right.
$$

temos que $f$ é equivalente a $h(x, y)=\left(x, a_{2} x^{2}+b_{2} x y+c_{2} y^{2}+R_{2}, a_{3} x^{2}+b_{3} x y+c_{3} y^{2}+R_{3}\right)$, finalmente efetuando a seguinte mudança de coordenadas na meta:

$$
\left\{\begin{array}{l}
U=u \\
V=v-a_{2} u^{2} \\
W=w-a_{3} u^{3}
\end{array}\right.
$$

temos que $f$ é equivalente a $\left(x, b_{1} x y+c_{1} y^{2}+R_{2}, b_{2} x y+c_{2} y^{2}+R_{3}\right)$.

Para demonstrar a segunda afirmação, observemos que se $j^{1} f \pitchfork \Sigma^{1}$ então

$$
d_{(0,0)}\left(j^{1} f\right)\left(\mathbb{R}^{2}\right)+T_{(0,0)} \Sigma^{1}=J^{1}(2,3),
$$


mas $\Sigma^{1}$ é uma subvariedade diferenciável de $J(2,3)$ com codimensão 2 , assim podemos calcular seu espaço tangente em $(0,0)$ e teremos:

$$
T_{(0,0)} \Sigma^{1}=[(1,0,0,0,0,0),(0,1,0,0,0,0),(0,0,1,0,0,0),(0,0,0,1,0,0)]
$$

por outro lado,

$$
d_{(0,0)}\left(j^{1} f\right)\left(\mathbb{R}^{2}\right)=\left[\left(0,0,0,0, b_{1}, b_{2}\right),\left(0, b_{1}, b_{2}, 0,2 c_{1}, 2 c_{2}\right)\right] .
$$

Como $d_{(0,0)}\left(j^{1} f\right)\left(\mathbb{R}^{2}\right)$ deve gerar os 2 vetores que faltam em $T_{(0,0)} \Sigma^{1}$, para uma base de $\mathbb{R}^{6}$, temos que $c_{2} b_{1}-c_{1} b_{2} \neq 0$.

Considerando a mudança de coordenadas

$$
\left\{\begin{array}{l}
X=x \\
Y=\alpha_{1}\left(b_{1} x y+c_{1} y^{2}\right)+\beta_{1}\left(b_{2} x y+c_{2} y^{2}\right) \\
Z=\alpha_{2}\left(b_{1} x y+c_{1} y^{2}\right)+\beta_{2}\left(b_{2} x y+c_{2} y^{2}\right)
\end{array}\right.
$$

onde $\alpha_{1}=\frac{c_{2}}{b_{1} c_{2}-b_{2} c_{1}}, \beta_{1}=\frac{-c_{1}}{b_{1} c_{2}-b_{2} c_{1}}, \alpha_{2}=\frac{b_{2}}{b_{2} c_{1}-b_{1} c_{2}}$ e $\beta_{2}=\frac{-b_{1}}{b_{2} c_{1}-b_{1} c_{2}}$, temos que $f$ é equivalente a $\left(x, x y+R_{2}, y^{2}+R_{3}\right)$, onde usamos a mesma notação $R_{2}$ e $R_{3}$ para os novos termos de ordem maior ou igual a 3 .

Entretanto pela proposição 2.8.6 temos que o guarda-chuva de Whitney é 2-determinado $\operatorname{logo} f$ é equivalente a $\left(x, x y, y^{2}\right)$.

Em seu artigo [27], Whitney definiu aplicações semi-regulares, que recordaremos a seguir.

Definição 3.1.10 Sejam $M$ uma 2-variedade e $f: M^{2} \rightarrow \mathbb{R}^{3}$ uma aplicação. Dizemos que $f$ é semi-regular se é de classe $C^{12}$ (pelo menos), própria e para cada $p \in M$ ou $f$ é regular em $p$ ou $\frac{\partial f}{\partial x}(p)=0$ e os vetores $\frac{\partial f}{\partial y}(p), \frac{\partial^{2} f}{\partial x \partial y}(p), \frac{\partial^{2} f}{\partial x^{2}}(p)$ são $L I$.

Observação 3.1.11 Nestas dimensões, para aplicações $C^{\infty}, f \pitchfork \Sigma^{i}$ significa que $f$ é semi-regular.

Teorema 3.1.12 Seja $f \in C^{\infty}\left(M^{2}, \mathbb{R}^{3}\right)$, com $M$ compacta ou $f$ própria, então $f$ é estável se, e somente se:

i) $f$ é semi-regular;

ii) $f: M-\Sigma(f) \rightarrow \mathbb{R}^{3}$ é uma imersão com cruzamento normal.

Demonstração: Suponha que $f$ seja estável então pelo teorema 3.1.1, $f$ é transversal a $\Sigma^{i}$, para todo $i$, assim pela observação anterior vale $(i)$. Por outro lado, se retiramos do domínio da $f$ os pontos de $\Sigma(f)$ então pelo teorema 3.1 .7 vale $(i i)$. 
A volta é uma consequência do teorema 2.5.6. Com efeito, as aplicações que satisfazem (i) e (ii) são infinitesimalmente estáveis (ver Teorema 3.1.9) e portanto, estáveis.

Colorário 3.1.13 O conjunto das aplicações estáveis é denso no conjunto das aplicações próprias de $M^{2}$ em $\mathbb{R}^{3}$.

Demonstração: Segundo o teorema e as observações anteriores, $f$ é estável então $f \pitchfork \Sigma^{i}$, para todo $i$, logo pelo teorema da transversalidade de Thom, o conjunto das aplicações com esta propriedade é denso em $C^{\infty}\left(M^{2}, \mathbb{R}^{3}\right)$. 


\section{Capítulo 4}

\section{Singularidades estáveis de superfícies regradas}

Neste capítulo discutimos o teorema central deste trabalho (Teorema 4.2.3). Com base no artigo [12], de S. Izumiya, vamos mostrar que o guarda-chuva de Whitney é também a única singularidade que ocorre genericamente no conjunto das superfícies regradas.

\subsection{Desdobramentos}

Definição 4.1.1 Um desdobramento r-dimensional de $f_{0}:\left(\mathbb{R}^{n}, 0\right) \rightarrow\left(\mathbb{R}^{p}, 0\right)$ é um germe $F:\left(\mathbb{R}^{n} \times \mathbb{R}^{r}, 0\right) \rightarrow\left(\mathbb{R}^{p} \times \mathbb{R}^{r}, 0\right)$ dado por $F(x, u)=(f(x, u), u)$ onde $f(x, u)$ é um germe de uma família parametrizada $r$-dimensional com $f_{0}(x)=f(x, 0)$.

Esta definição depende do sistema de coordenadas de ambos os espaços $\left(\mathbb{R}^{n} \times \mathbb{R}^{r}, 0\right)$ e $\left(\mathbb{R}^{p} \times \mathbb{R}^{r}, 0\right)$. Precisamos de uma definição que não dependa. Assim temos:

Definição 4.1.2 Seja $f:\left(N, x_{0}\right) \rightarrow\left(P, y_{0}\right)$ um germe de aplicação entre variedades. Um desdobramento de $f$ é uma terna $(F, i, j)$ de germes de aplicações onde:

i) $i:\left(N, x_{0}\right) \rightarrow\left(N^{\prime}, x_{0}^{\prime}\right)$ e $j:\left(P, y_{0}\right) \rightarrow\left(P^{\prime}, y_{0}^{\prime}\right)$ são germes de imersões com $j \pitchfork F$ tais que $F \circ i=j \circ f$;

ii) $(i, f): N \rightarrow\left\{\left(x^{\prime}, y\right) \in N^{\prime} \times P / F\left(x^{\prime}\right)=j(y)\right\}$ é um difeomorfismo.

$A$ dimensão de $(F, i, j)$ como desdobramento é $\operatorname{dim} N^{\prime}$-dimN.

Proposição 4.1.3 As duas definições de desdobramento são equivalentes se $\left(N, x_{0}\right)=$ $\left(\mathbb{R}^{n}, 0\right),\left(P, y_{0}\right)=\left(\mathbb{R}^{p}, 0\right),\left(N^{\prime}, x_{0}^{\prime}\right)=\left(\mathbb{R}^{n} \times \mathbb{R}^{r}, 0 \times 0\right) e\left(P^{\prime}, y_{0}^{\prime}\right)=\left(\mathbb{R}^{p} \times \mathbb{R}^{r}, 0 \times 0\right)$, onde $r$ é a dimensão do desdobramento. 
Observação 4.1.4 Quando falamos que as definições são equivalentes estamos dizendo que dada F segundo a definição 4.1.1, então a terna $(F, i, j)$, com $i$ e $j$ inclusões canônicas, satisfaz a definição 4.1.2. Reciprocamente, dada uma terna $(F, i, j)$ satisfazendo à definição 4.1.2 então $F$ é $\mathcal{A}$-equivalente a uma função $F^{\prime}$ nas condições da definição 4.1.1.

Demonstração: $(4.1 .1 \Rightarrow 4.1 .2)$

Inicialmente, temos que as inclusões canônicas $i:\left(\mathbb{R}^{n}, 0\right) \rightarrow\left(\mathbb{R}^{n} \times \mathbb{R}^{r}, 0 \times 0\right) \mathrm{e}$ $j:\left(\mathbb{R}^{p}, 0\right) \rightarrow\left(\mathbb{R}^{p} \times \mathbb{R}^{r}, 0 \times 0\right)$ são germes de imersões. Mostremos então que a terna $(F, i, j)$ satisfaz à definição 4.1.2.

i) Como $F(x, u)=(f(x, u), u)$ temos que a matriz Jacobiana de $F$, na origem, é formada por uma matriz identidade no ultimo menor $r \times r$. Logo

$$
d_{0} j\left(\mathbb{R}^{p}\right)+d_{0 \times 0} F\left(\mathbb{R}^{n} \times \mathbb{R}^{r}\right)=\mathbb{R}^{p} \times \mathbb{R}^{r},
$$

portanto $j \pitchfork F$. E mais,

$$
F \circ i(x)=F(x, 0)=\left(f_{0}(x), 0\right)=j\left(f_{0}(x)\right)=j \circ f_{0}(x)
$$

ii) Temos agora que mostrar que

$$
\left(i, f_{0}\right): \mathbb{R}^{n} \rightarrow\left\{(x, u, y) \in \mathbb{R}^{n} \times \mathbb{R}^{r} \times \mathbb{R}^{p} / F(x, u)=j(y)\right\}
$$

é um difeomorfismo.

Para isso basta observar que

$$
\left\{(x, u, y) \in \mathbb{R}^{n} \times \mathbb{R}^{r} \times \mathbb{R}^{p} / F(x, u)=j(y)\right\}=\left\{\left(x, 0, f_{0}(x)\right) \in \mathbb{R}^{n} \times \mathbb{R}^{r} \times \mathbb{R}^{p}\right\}
$$

assim a diferencial de $\left(i, f_{0}\right)$ é uma bijeção entre $\mathbb{R}^{n}$ e este conjunto, portanto $\left(i, f_{0}\right)$ é difeomorfismo.

$$
(4.1 .2 \Rightarrow 4.1 .1)
$$

Seja $\left\{e_{1}, \ldots, e_{p+1}, \ldots, e_{p+r}\right\} \subset \mathbb{R}^{p} \times \mathbb{R}^{r}$ base canônica. Pelo teorema da forma local das imersões podemos considerar $j$ da seguinte maneira $j(x)=(x, 0)$, logo como $j \pitchfork F$ temos que os vetores $\left\{e_{p+1}, \ldots, e_{p+r}\right\} \subset \mathbb{R}^{p} \times \mathbb{R}^{r}$ estão na imagem da diferencial de $F$. Portanto o último menor de $F$ é invertível e existem difeomorfismos tais que $F$ é $\mathcal{A}$-equivalente a $F^{\prime}(x, u)=(g(x, u), u)$.

Por outro lado $F^{\prime}(x, 0)=F^{\prime} \circ i(x)=j \circ f_{0}(x)=\left(f_{0}(x), 0\right)$, assim $g(x, 0)=f_{0}(x)$. Portanto $g(x, u)$ é um germe de uma família parametrizada $r$-dimensional com $g(x, 0)=$ $f_{0}(x)$.

Concluímos $F$ é $\mathcal{A}$-equivalente a um desdobramento segunda a definição 4.1.1. 
Definição 4.1.5 Dois desdobramentos $F_{1}$ e $F_{2}$ a $r$ parâmetros de $f$ são equivalentes quando existe um desdobramento de $f$ a $r$ parâmetros $I$ da identidade tal que $F_{1}=I \circ F_{2}$.

Definição 4.1.6 Sejam $F$ um desdobramento a $r$ parâmetros de $f$ e $H:\left(\mathbb{R}^{s}, 0\right) \rightarrow$ $\left(\mathbb{R}^{r}, 0\right)$ um germe. Dizemos que o desdobramento a s parâmetros de $f$ dado por $G=F \circ H$ é induzido por $H$.

Definição 4.1.7 Sejam $F$ e $G$ desdobramentos de $f$ a r e a s parâmetros respectivamente. Um morfismo de $F$ em $G$ é um par $(H, I)$ com I um desdobramento a r parâmetros da identidade e $H$ uma mudança de parâmetros para os quais F é equivalente ao desdobramento $G \circ H$. Quando $r=s$ e $H$ é invertível chamamos o morfismo de isomorfismo.

Definição 4.1.8 Um desdobramento $F$ de $f$ é dito versal quando para todo desdobramento $G$ de $f$ existe um morfismo de $F$ em $G$. Um desdobramento versal com o número minimo de parâmetros é denominado mini-versal.

Teorema 4.1.9 Uma aplicação f é estável quando ela é seu próprio desdobramento versal.

Demonstração: Ver [7].

Agora podemos lançar mão de um lema bastante simples para olharmos uma superfície regrada $F: I \times J \subset \mathbb{R}^{2} \rightarrow \mathbb{R}^{3}$ como desdobramento 1-dimensional de uma aplicação $f: \mathbb{R} \rightarrow \mathbb{R}^{2}$.

Lema 4.1.10 Seja $F:\left(\mathbb{R}^{2}, 0\right) \rightarrow\left(\mathbb{R}^{3}, 0\right)$ um germe de aplicação com $F(t, u)=$ $\left(F_{1}(t, u), F_{2}(t, u), F_{3}(t, u)\right)$. Suponha que $\frac{\partial F_{3}}{\partial u}(0) \neq 0$ e, portanto pelo Teorema da Função Implícita, existe uma vizinhança da origem tal que $F_{3}^{-1}(0)=\left\{(t, g(t)) \in \mathbb{R}^{2} / t \in(\mathbb{R}, 0)\right\}$. Consideremos os germes de imersões $i(t)=(t, g(t))$ e $j:\left(\mathbb{R}^{2}, 0\right) \rightarrow\left(\mathbb{R}^{3}, 0\right), j\left(y_{1}, y_{2}\right)=$ $\left(y_{1}, y_{2}, 0\right)$ e um germe de aplicação $f:(\mathbb{R}, 0) \rightarrow\left(\mathbb{R}^{2}, 0\right), f(t)=\left(F_{1}(t, g(t)), F_{2}(t, g(t))\right)$. Então $(F, i, j)$ é um desdobramento 1-dimensional de $f$.

Demonstração: Primeiramente temos

$$
F \circ i(t)=F(t, g(t))=\left(F_{1}(t, g(t)), F_{2}(t, g(t)), 0\right)=(f(t), 0)=j \circ f(t)
$$

Como $\frac{\partial F_{3}}{\partial u}(0) \neq 0$ e $T(I m j)=<(1,0,0),(0,1,0)>$, então $d F\left(\mathbb{R}^{2}\right)+T(I m j)=\mathbb{R}^{3}$ e portanto $F \pitchfork j$. 
Finalmente para $(i, f):(\mathbb{R}, 0) \rightarrow\left(\mathbb{R}^{2} \times \mathbb{R}^{2}, 0\right)$ vale que

$$
(i, f)(t)=\left(t, g(t), F_{1}(t, g(t)), F_{2}(t, g(t))\right)
$$

mas

$\left\{(x, y) \in \mathbb{R}^{2} \times \mathbb{R}^{2} / F(x)=j(y)\right\}=\left\{\left(t, g(t), F_{1}(t, g(t)), F_{2}(t, g(t))\right) \in \mathbb{R}^{2} \times \mathbb{R}^{2} / t \in(\mathbb{R}, 0)\right\}$

$\operatorname{assim}(i, f)$ é difeomorfismo.

Para o que segue usaremos a seguinte notação, $\varepsilon_{x, y}$ denotará o conjunto dos germes de aplicações $f:\left(\mathbb{R}^{n} \times \mathbb{R}^{r}, 0 \times 0\right) \rightarrow \mathbb{R}^{p}$ de classe $C^{\infty}$ onde $x=\left(x_{1}, \ldots, x_{n}\right)$ denota as variáveis em $\mathbb{R}^{n}$ e $y=\left(y_{1}, \ldots, y_{r}\right)$ as de $\mathbb{R}^{r}$. De maneira análoga definimos $\varepsilon_{x}, \varepsilon_{y}, m_{x}$ e $\varepsilon_{x, y}^{0}$.

Definição 4.1.11 Consideremos agora o ideal gerado por $y_{1}, \ldots, y_{r}$ com coeficientes em $\varepsilon_{x, y}$ e o denotemos por $\langle y\rangle$ então definimos $M_{0}=\frac{M}{\langle y>. M}$ onde $M$ é um $\varepsilon_{x, y}$-módulo de tipo finito.

Observação 4.1.12 Note que $M_{0}$ é um $\varepsilon_{x}$-módulo de tipo finito.

Teorema 4.1.13 (Teorema de Preparação de Malgrange) Seja $f:\left(\mathbb{R}^{n}, 0\right) \rightarrow\left(\mathbb{R}^{p}, 0\right)$ germe de aplicação $C^{\infty}$ e $M$ um $\varepsilon_{n}$-módulo finitamente gerado. Então $M$ é um $\varepsilon_{p}$-módulo (via $f^{*}$ ) finitamente gerado se, e somente se, $\frac{M}{f^{*}\left(m_{p}\right) \cdot M}$ é um espaço vetorial de dimensão finita.

Demonstração: Ver [9].

Teorema 4.1.14 Seja $M$ um $\varepsilon_{x, y}$-módulo de tipo finito com geradores $\left\{m_{1}, \ldots, m_{k}\right\}$. Seja $N$ um sub-módulo de $M$. As seguintes condições são equivalentes:

i) $\bar{N}+\mathbb{R}\left\{m_{1,0}, \ldots, m_{k, 0}\right\}=M_{0}$, onde $\bar{N}$ é a projeção de $N$ em $M_{0}$.

ii) $N+\varepsilon_{y}\left\{m_{1}, \ldots, m_{k}\right\}=M$, onde $m_{i} \in M$ é projetado sobre $m_{i, 0} \in M_{0}$.

Demonstração: Ver [15].

Definição 4.1.15 Para todo germe $f:\left(\mathbb{R}^{n} \times \mathbb{R}^{r}, 0 \times 0\right) \rightarrow\left(\mathbb{R}^{p}, 0\right)$ definimos o germe $j_{1}^{k} f:\left(\mathbb{R}^{n} \times \mathbb{R}^{r}, 0 \times 0\right) \rightarrow J^{k}(n, p)$ dado por $j_{1}^{k} f\left(x_{0}, u_{0}\right)=j^{k} f_{u_{0}}\left(x_{0}\right)$ onde $f_{u}(x)=f(x, u)$ e $j^{k} f_{u_{0}}(x)=j^{k}\left(f_{u_{0}}\left(x+x_{0}\right)\right)(0)$. 
Lema 4.1.16 Seja $K^{k}\left(j_{1}^{k} f_{0}(0)\right)$ a K-órbita de $j_{1}^{k} f_{0}(0)$ em $J^{k}(n, p)$. Nas condições da definição anterior, $j_{1}^{k} f$ é transversal a $K^{k}\left(j_{1}^{k} f_{0}(0)\right)$, se, e somente se,

$$
\varepsilon_{n, p}=\left\langle\frac{\partial f_{0}}{\partial x_{1}}, \ldots, \frac{\partial f_{0}}{\partial x_{n}}\right\rangle_{\varepsilon_{n}}+f_{0}^{*}\left(m_{p}\right) \varepsilon_{n, p}+\left\langle\frac{\partial f}{\partial u_{1}}(x, 0), \ldots, \frac{\partial f}{\partial u_{r}}(x, 0), e_{1}, \ldots, e_{p}\right\rangle_{\mathbb{R}}
$$

onde $e_{i}=(0, \ldots, 1, \ldots, 0) \in \mathbb{R}^{p}$.

Demonstração: Temos por definição que $j_{1}^{k} f \pitchfork K^{k}\left(j^{k} f_{0}(0)\right)$ se, e somente se,

$$
d_{p}\left(j_{1}^{k} f\right)\left(\mathbb{R}^{n} \times \mathbb{R}^{p}\right)+T_{q} K^{k}\left(j^{k} f_{0}(0)\right)=J^{k}(n, p)
$$

para todo $p \in\left(\mathbb{R}^{n} \times \mathbb{R}^{p}, 0 \times 0\right) \operatorname{com} q=j_{1}^{k} f(p) \in K^{k}\left(j^{k} f_{0}(0)\right)$.

Entretanto em nosso caso estamos lidando com germes na origem isso nos permite avaliar esta igualdade apenas em $(0 \times 0) \in \mathbb{R}^{n} \times \mathbb{R}^{p}$.

Calculemos então $d_{(0,0)}\left(j_{1}^{k} f\right)\left(\mathbb{R}^{n} \times \mathbb{R}^{p}\right)$. Nos valendo de um abuso de notação temos, para $1 \leq i \leq n$ :

$$
\begin{gathered}
\frac{\partial j_{1}^{k} f}{\partial x_{i}}(0,0)=\lim _{\|t\| \rightarrow 0} \frac{\left\|j_{1}^{k} f\left((0,0)+t\left(w_{i}, 0\right)\right)-j_{1}^{k} f(0,0)\right\|}{\|t\|}=\lim _{\|t\| \rightarrow 0} \frac{\left\|j^{k} f_{0}\left(0+t w_{i}\right)-j^{k} f_{0}(0)\right\|}{\|t\|}= \\
\lim _{\|t\| \rightarrow 0} \frac{\left\|j^{k}\left(f_{0}\left(x+t w_{i}\right)\right)(0)-j^{k}\left(f_{0}(x)\right)(0)\right\|}{\|t\|}=j^{k}\left(\lim _{\|t\| \rightarrow 0} \frac{\left\|f_{0}\left(x+t w_{i}\right)-f_{0}(0)\right\|}{\|t\|}\right)(0)= \\
j^{k}\left(\frac{\partial f_{0}}{\partial x_{i}}(x)\right)(0),
\end{gathered}
$$

onde $\left\{w_{i} \in \mathbb{R}^{n} / 1 \leq i \leq n\right\}$ é a base canônica de $\mathbb{R}^{n}$.

Agora para $1 \leq i \leq r$ temos

$$
\begin{gathered}
\frac{\partial j_{1}^{k} f}{\partial u_{i}}(0,0)=\lim _{\|t\| \rightarrow 0} \frac{\left\|j_{1}^{k} f\left((0,0)+t\left(0, v_{i}\right)\right)-j_{1}^{k} f(0,0)\right\|}{\|t\|}=\lim _{\|t\| \rightarrow 0} \frac{\left\|j^{k} f_{t v_{i}}(0)-j^{k} f_{0}(0)\right\|}{\|t\|}= \\
\lim _{\|t\| \rightarrow 0} \frac{\left\|j^{k}\left(f_{t v_{i}}(x)\right)(0)-j^{k}\left(f_{0}(x)\right)(0)\right\|}{\|t\|}=j^{k}\left(\lim _{\|t\| \rightarrow 0} \frac{\left\|f_{t v_{i}}(x)-f_{0}(x)\right\|}{\|t\|}\right)(0)= \\
j^{k}\left(\lim _{\|t\| \rightarrow 0} \frac{\left\|f\left(x, t v_{i}\right)-f(x, 0)\right\|}{\|t\|}\right)(0)=j^{k}\left(\frac{\partial f}{\partial u_{i}}(x, 0)\right)(0),
\end{gathered}
$$

onde $\left\{v_{i} \in \mathbb{R}^{n} / 1 \leq i \leq r\right\}$ é a base canônica de $\mathbb{R}^{r}$.

Portanto,

$d_{(0,0)}\left(j_{1}^{k} f\right)\left(\mathbb{R}^{n} \times \mathbb{R}^{p}\right)=j^{k}\left(\left\langle\frac{\partial f_{0}}{\partial x_{1}}(x), \ldots, \frac{\partial f_{0}}{\partial x_{n}}(x)\right\rangle_{\mathbb{R}}+\left\langle\frac{\partial f}{\partial u_{1}}(x, 0), \ldots, \frac{\partial f}{\partial u_{r}}(x, 0)\right\rangle_{\mathbb{R}}\right)(0)$.

Por outro lado sabemos que

$$
T_{j^{k} f_{0}(0)} K^{k}\left(j^{k} f_{0}(0)\right)=j^{k}\left(\left\langle\frac{\partial f_{0}}{\partial x_{1}}(x), \ldots, \frac{\partial f_{0}}{\partial x_{n}}(x)\right\rangle_{\varepsilon_{n}}+f^{*}\left(m_{p}\right) \varepsilon_{n, p}\right)(0) .
$$


Assim $j_{1}^{k} f \pitchfork K^{k}\left(j^{k} f_{0}(0)\right)$ se, e somente se,

$m_{n} \varepsilon_{n, p}=\left\langle\frac{\partial f_{0}}{\partial x_{1}}(x), \ldots, \frac{\partial f_{0}}{\partial x_{n}}(x)\right\rangle_{\varepsilon_{n}}+f^{*}\left(m_{p}\right) \varepsilon_{n, p}+\left\langle\frac{\partial f}{\partial u_{1}}(x, 0), \ldots, \frac{\partial f}{\partial u_{r}}(x, 0)\right\rangle_{\mathbb{R}}+m_{n}^{k+1} \varepsilon_{n, p}$.

Mas esta igualdade é equivalente à

$\varepsilon_{n, p}=$

$\left\langle\frac{\partial f_{0}}{\partial x_{1}}(x), \ldots, \frac{\partial f_{0}}{\partial x_{n}}(x)\right\rangle_{\varepsilon_{n}}+f^{*}\left(m_{p}\right) \varepsilon_{n, p}+\left\langle\frac{\partial f}{\partial u_{1}}(x, 0), \ldots, \frac{\partial f}{\partial u_{r}}(x, 0), e_{1}, \ldots, e_{p}\right\rangle_{\mathbb{R}}+m_{n}^{k+1} \varepsilon_{n, p}$,

onde $\left\{e_{i} \in \mathbb{R}_{p} / 1 \leq i \leq p\right\}$ é base de $\mathbb{R}^{p}$.

Uma observação a ser feita é que os cálculos efetuados até agora não dependeram de $k$. Portanto suporemos agora que $k+1>p+r$. Seja $M=\left\langle\frac{\partial f_{0}}{\partial x_{1}}(x), \ldots, \frac{\partial f_{0}}{\partial x_{n}}(x)\right\rangle_{\varepsilon_{n}}+f^{*}\left(m_{p}\right) \varepsilon_{n, p}$ o $\varepsilon_{n}$-submódulo de $\varepsilon_{n, p}$ e consideremos a seguinte cadeia encaixante de conjuntos

$$
\varepsilon_{n, p} \supset m_{n} \varepsilon_{n, p}+M \supset \ldots \supset m_{n}^{k} \varepsilon_{n, p}+M \supset \ldots
$$

Seja $c_{i}$ a codimensão de $m_{n}^{i+1}+M$ em $m_{n}^{i}+M$, então valem as seguintes condições:

a) se $c_{i}=0$ então $c_{i+1}=c_{i+2}=\ldots=0$;

b) $\operatorname{dim} \frac{\varepsilon_{n, p}}{m_{n}^{i} \varepsilon_{n, p}+M}=c_{1}+\ldots+c_{i}$.

Entretanto pela igualdade anterior temos que $\operatorname{dim} \frac{\varepsilon_{n, p}}{m_{n}^{k+1} \varepsilon_{n, p}+M}=\leq r+p \operatorname{logo} c_{1}+\ldots+$ $c_{k+1} \leq r+p$ como $k+1>p+r$ temos que $c_{k+1}=0 \operatorname{logo} m_{n}^{k+1} \varepsilon_{n, p}+M \subset m_{n}^{k+2} \varepsilon_{n, p}+M$ e pelo lema de Nakayama $m_{n}^{k+1} \varepsilon_{n, p} \subset M$.

Finalmente, dado estas observações, temos que

$$
\varepsilon_{n, p}=\left\langle\frac{\partial f_{0}}{\partial x_{1}}(x), \ldots, \frac{\partial f_{0}}{\partial x_{n}}(x)\right\rangle_{\varepsilon_{n}}+f^{*}\left(m_{p}\right) \varepsilon_{n, p}+\left\langle\frac{\partial f}{\partial u_{1}}(x, 0), \ldots, \frac{\partial f}{\partial u_{r}}(x, 0), e_{1}, \ldots, e_{p}\right\rangle_{\mathbb{R}} .
$$

Lema 4.1.17 Seja $F:\left(\mathbb{R}^{n} \times \mathbb{R}^{r}, 0 \times 0\right) \rightarrow\left(\mathbb{R}^{p} \times \mathbb{R}^{r}, 0 \times 0\right)$ um desdobramento de $f_{0}$ da forma $F(x, u)=(f(x, u), u)$. Se $j_{1}^{k}$ é transversal a $K^{k}\left(j_{1}^{k} f_{0}(0)\right)$ para $k$ suficientemente grande, então F é infinitesimalmente estável.

Demonstração: Consideremos a projeção canônica $\pi: \varepsilon_{n+r, p} \rightarrow \varepsilon_{n, p}$. Como para toda $f \in \varepsilon_{n+r, p}$ podemos escrever $f(x, u)=f(x, 0)+u g(x, u)$ bastando apenas fazer a série de Taylor de $f$, segundo a variável $u$ em torno do ponto 0 , então concluímos que $\pi$ é sobrejetora.

Assim usando o lema anterior temos $\varepsilon_{n+r, p}=\pi^{-1}\left(\varepsilon_{n, p}\right)=$ $\left\langle\frac{\partial f_{0}}{\partial x_{1}}(x), \ldots, \frac{\partial f_{0}}{\partial x_{n}}(x)\right\rangle_{\varepsilon_{n+r}}+f_{0}^{*}\left(m_{p}\right) \varepsilon_{n+r, p}+\left\langle\frac{\partial f}{\partial u_{1}}(x, 0), \ldots, \frac{\partial f}{\partial u_{r}}, e_{1}, \ldots, e_{p}\right\rangle_{\varepsilon_{r}}+m_{r} \varepsilon_{n+r, p}$. 
Aplicando o teorema 4.1.14 para $M=\varepsilon_{n+r, p}$ que é um $\varepsilon_{n+r}$-módulo de tipo finito,

$$
N=\left\langle\frac{\partial f_{0}}{\partial x_{1}}(x), \ldots, \frac{\partial f_{0}}{\partial x_{n}}(x)\right\rangle_{\varepsilon_{n+r}}+f_{0}^{*}\left(m_{p}\right) \varepsilon_{n+r, p}
$$

que é um $\varepsilon_{r}$-submódulo de $M$ e

$$
M_{0}=\frac{\varepsilon_{n+r, p}}{m_{r} \varepsilon_{n+r, p}}
$$

temos

$$
\varepsilon_{n+r, p}=\left\langle\frac{\partial f_{0}}{\partial x_{1}}(x), \ldots, \frac{\partial f_{0}}{\partial x_{n}}(x)\right\rangle_{\varepsilon_{n+r}}+f_{0}^{*}\left(m_{p}\right) \varepsilon_{n+r, p}+\left\langle\frac{\partial f}{\partial u_{1}}(x, 0), \ldots, \frac{\partial f}{\partial u_{r}}, e_{1}, \ldots, e_{p}\right\rangle_{\varepsilon_{r}} .
$$

Logo para todo $\xi=\left(\xi_{1}, \xi_{2}\right) \in \varepsilon_{n+r, p+r}=\varepsilon_{n+r, p} \times \varepsilon_{n+r, r}$, existem $\lambda_{i}, \eta_{i} \in \varepsilon_{n+r}$ e $\mu_{i}, \zeta_{i} \in \varepsilon_{r}$ tais que

$$
\xi_{1}=\sum_{i=1}^{n} \lambda_{i} \frac{\partial f_{0}}{\partial x_{i}}+\sum_{i=1}^{p} \eta_{i} f_{0, i}+\sum_{i=1}^{r} \mu_{i} \frac{\partial f}{u_{i}}+\sum_{i=1}^{p} \zeta_{i} e_{i}
$$

Portanto, temos

$$
\begin{gathered}
\left(\xi_{1}, 0\right)=\sum_{i=1}^{n} \lambda_{i} \frac{\partial F}{\partial x_{i}}+\sum_{i=1}^{p} \eta_{i}\left(f_{i}, 0\right)+\sum_{i=1}^{r} \mu_{i}\left(\frac{\partial f}{u_{i}}, 0\right)+\sum_{i=1}^{p} \zeta_{i}\left(e_{i}, 0\right)= \\
\sum_{i=1}^{n} \lambda_{i} \frac{\partial F}{\partial x_{i}}+\sum_{i=1}^{p} \eta_{i}\left(f_{i}, 0\right)+\sum_{i=1}^{r} \mu_{i}\left(\frac{\partial f}{u_{i}}, 0\right)-\sum_{i=1}^{n} \lambda_{i} \frac{\partial F}{\partial u_{i}}+\sum_{i=1}^{n} \lambda_{i} \frac{\partial F}{\partial u_{i}}+\sum_{i=1}^{p} \zeta_{i}\left(e_{i}, 0\right)= \\
\sum_{i=1}^{n} \lambda_{i} \frac{\partial F}{\partial x_{i}}+\sum_{i=1}^{n} \lambda_{i} \frac{\partial F}{\partial u_{i}}+\sum_{i=1}^{p} \eta_{i}\left(f_{i}, 0\right)+\sum_{i=1}^{p} \zeta_{i}\left(e_{i}, 0\right)-\sum_{i=1}^{r} \mu_{i}\left(0, v_{i}\right),
\end{gathered}
$$

onde $\left\{v_{i} \in \mathbb{R}^{r} / 1 \leq i \leq r\right\}$ é a base canônica de $\mathbb{R}^{r}$.

Como $\mu_{i}, \zeta_{i} \in \varepsilon_{r}$ temos que $\sum_{i=1}^{p} \zeta_{i}\left(e_{i}, 0\right)-\sum_{i=1}^{r} \mu_{i}\left(0, v_{i}\right) \in F^{*} \varepsilon_{p+r, p+r}$. Isto implica que

$$
\left(\xi_{1}, 0\right) \in\left\langle\frac{\partial F}{\partial x_{1}}, \ldots, \frac{\partial F}{\partial x_{n}}, \frac{\partial F}{\partial u_{1}}, \ldots, \frac{\partial F}{\partial u_{r}}\right\rangle_{\varepsilon_{n+r}}+F^{*} \varepsilon_{p+r, p+r}+F^{*}\left(m_{p+r}\right) \varepsilon_{n+r, p+r} .
$$

Por outro lado temos que

$$
\left(0, \xi_{2}\right)=\sum_{i=1}^{r} \xi_{2, i} \frac{\partial F}{\partial u_{i}}-\sum_{i=1}^{r} \xi_{2, i}\left(\frac{\partial f}{\partial u_{i}}, 0\right)
$$

Aplicando o mesmo argumento usado para $\left(\xi_{1}, 0\right)$ temos que

$$
\left(0, \xi_{2}\right) \in\left\langle\frac{\partial F}{\partial x_{1}}, \ldots, \frac{\partial F}{\partial x_{n}}, \frac{\partial F}{\partial u_{1}}, \ldots, \frac{\partial F}{\partial u_{r}}\right\rangle_{\varepsilon_{n+r}}+F^{*} \varepsilon_{p+r, p+r}+F^{*}\left(m_{p+r}\right) \varepsilon_{n+r, p+r} .
$$


Concluímos então que

$$
\varepsilon_{n+r, p+r}=\left\langle\frac{\partial F}{\partial x_{1}}, \ldots, \frac{\partial F}{\partial x_{n}}, \frac{\partial F}{\partial u_{1}}, \ldots, \frac{\partial F}{\partial u_{r}}\right\rangle_{\varepsilon_{n+r}}+F^{*} \varepsilon_{p+r, p+r}+F^{*}\left(m_{p+r}\right) \varepsilon_{n+r, p+r} .
$$

Aplicando novamente o teorema 4.1.14 temos

$$
\varepsilon_{n+r, p+r}=\left\langle\frac{\partial F}{\partial x_{1}}, \ldots, \frac{\partial F}{\partial x_{n}}, \frac{\partial F}{\partial u_{1}}, \ldots, \frac{\partial F}{\partial u_{r}}\right\rangle_{\varepsilon_{n+r}}+F^{*} \varepsilon_{p+r, p+r} .
$$

\subsection{Classificação Genérica}

Provaremos agora que, para curvas genéricas $(\gamma, \delta)$, o germe de uma superfície regrada $F_{(\gamma, \delta)}$ em todo ponto, é infinitamente estável.

Os pontos singulares de uma superfície regrada $F_{(\gamma, \delta)}$ são dados pela condição posto $\left(\gamma^{\prime}(t)\right.$ $\left.+u \delta^{\prime}(t), \delta(t)\right)<2$ que é equivalente à condição dos vetores $\gamma^{\prime}(t)+u \delta^{\prime}(t)$ e $\delta(t)$ serem paralelos. Finalmente como $\delta(t) \neq 0$ temos que posto $\left(\gamma^{\prime}(t)+u \delta^{\prime}(t), \delta(t)\right)=1$.

Agora consideremos o parâmetro $u$ (isto é, o parâmetro ao longo da geratriz) da superfície regrada como o parâmetro de um desdobramento unidimensional. Para todo $(\gamma, \delta): I \rightarrow \mathbb{R}^{3} \times S^{2} \operatorname{com} \delta^{\prime}(t) \neq 0$ onde $\gamma(t)=\left(\gamma_{1}(t), \gamma_{2}(t), \gamma_{3}(t)\right)$ e $\delta(t)=\left(\delta_{1}(t), \delta_{2}(t), \delta_{3}(t)\right)$ temos

$$
F_{(\gamma, \delta)}(t, u)=\left(\gamma_{1}(t)+u \delta_{1}(t), \gamma_{2}(t)+u \delta_{2}(t), \gamma_{3}(t)+u \delta_{3}\right) .
$$

Para todo $\left(t_{0}, u_{0}\right) \in I \times J$ fixo com $\delta_{3}\left(t_{0}\right) \neq 0\left(\right.$ se $\delta_{3}\left(t_{0}\right)=0$ podemos escolher $\delta_{1}$ ou $\delta_{2}$ pois $\delta(t) \neq 0$ para todo $\left.t \in I\right)$ definimos $U_{3}=\left\{t \in I / \delta_{3}(t) \neq 0\right\} \subset I$, assim definido $U_{3}$ é não vazio.

Definimos $g_{3}(t)=-\frac{\left(\gamma_{3}(t)-y_{0}\right)}{\delta_{3}(t)}$ para $t \in U_{3}$ e $y_{0}=\gamma_{3}\left(t_{0}\right)+u_{0} \delta_{3}\left(t_{0}\right)$. Logo $F$ toma a seguinte forma:

$$
F_{(\gamma, \delta)}(t, u)=\gamma(t)+g_{3}(t) \delta(t)+\left(u-g_{3}(t)\right) \delta(t)
$$

e fazendo a mudança $T=t$ e $U=u-g_{3}(t)$ temos

$$
\widetilde{F}_{(\gamma, \delta)}(T, U)=\gamma(T)+g_{3}(T) \delta(T)+U \delta(T) .
$$

Pelo lema 4.1.10 o germe $\widetilde{F}_{(\gamma, \delta)}(T, U)$ em $\left(t_{0}, 0\right)$ é um desdobramento unidimensional de $\pi_{3} \circ \widetilde{F}_{(\gamma, \delta)}(T, 0)=\left(\gamma_{1}(T)+g_{3}(T) \delta_{1}(T), \gamma_{2}(T)+g_{3}(T) \delta_{2}(T)\right)$, onde $\pi_{3}: \mathbb{R}^{3} \rightarrow \mathbb{R}^{2}$ é a projeção canônica $\pi_{3}\left(y_{1}, y_{2}, y_{3}\right)=\left(y_{1}, y_{2}\right)$.

Lema 4.2.1 Sejam $W$ uma subvariedade de $J^{k}(1,2)$ e $K$ um subconjunto compacto de $W$ então $O_{W, K}=\left\{g \in C^{\infty}\left(X, J^{k}(1,2)\right) / g \pitchfork W\right.$ em $\left.K\right\}$ é um aberto de $C^{\infty}\left(X, J^{k}(1,2)\right)$. 
Demonstração: Ver [9], lema 4.14.

Lema 4.2.2 Seja $W \subset J^{k}(1,2)$ uma subvariedade. Para todo germe $\delta: I \rightarrow S^{2}$ fixo com $\delta^{\prime}(t) \neq 0$ e todo ponto $\left(t_{0}, u_{0}\right) \in I \times J$ fixo com $\delta_{3}\left(t_{0}\right) \neq 0$ o conjunto

$$
T_{W,\left(t_{0}, u_{0}\right)}=\left\{\gamma \in C^{\infty}\left(I, \mathbb{R}^{3}\right) / j_{1}^{k} \pi_{3} \circ \widetilde{F}_{(\gamma, \delta)} \pitchfork W \text { em }\left(t_{0}, u_{0}\right)\right\}
$$

é um subconjunto residual de $C^{\infty}\left(I, \mathbb{R}^{3}\right) \times\{\delta\}$. Aqui consideramos que $C^{\infty}\left(I, \mathbb{R}^{3} \times S^{2}\right)=$ $C^{\infty}\left(I, \mathbb{R}^{3}\right) \times C^{\infty}\left(I, S^{2}\right)$ e a topologia de $C^{\infty}\left(I, \mathbb{R}^{3}\right) \times\{\delta\}$ é a induzida.

Demonstração: Seja $\left\{K_{j}\right\}_{j=1}^{\infty}$ um conjunto enumerável de abertos cobrindo $W$ tais que $\bar{K}_{j}$ é compacto. Definimos

$$
T_{W,\left(t_{0}, u_{0}\right), K_{j}}=\left\{\gamma \in C^{\infty}\left(I, \mathbb{R}^{3}\right) / j_{1}^{k} \widetilde{\pi}_{3} \circ \widetilde{F}_{\gamma, \delta} \pitchfork W \operatorname{com} j_{1}^{k} \widetilde{\pi}_{3} \circ \widetilde{F}_{\gamma, \delta}\left(t_{0}, u_{0}\right) \in \bar{K}_{j}\right\}
$$

Mostremos que $T_{W,\left(t_{0}, u_{0}\right), K_{j}}$ é aberto. Consideremos as aplicações contínuas

$$
\widehat{j}^{k}: C^{\infty}\left(U_{3}, \mathbb{R}^{3}\right) \rightarrow C^{\infty}\left(U_{3} \times J, J^{k}(1,2)\right)
$$

definida por $\widehat{j}^{k}(\gamma)=j^{k} \widetilde{\pi}_{3} \circ \widetilde{F}_{(\gamma, \delta)}$, e a aplicação restrição

$$
\operatorname{res}_{U_{3}}: C^{\infty}\left(I, \mathbb{R}^{3}\right) \rightarrow C^{\infty}\left(U_{3}, \mathbb{R}^{3}\right)
$$

Como $O_{W, K_{j}}=\left\{g \in C^{\infty}\left(U_{3} \times J, J^{k}(1,2)\right) / g \pitchfork W\right.$ em $\left.\left(t_{0}, u_{0}\right) \operatorname{com} g\left(t_{0}, u_{0}\right) \in K_{j}\right\}$ é aberto temos que $T_{W,\left(t_{0}, u_{0}\right), K_{j}}=\left(\operatorname{res}_{U_{3}}\right)^{-1} \circ\left(\widehat{j}^{k}\right)^{-1}\left(O_{W, K_{j}}\right)$ também é aberto.

Se mostrarmos que $T_{W,\left(t_{0}, u_{0}\right), K_{j}}$ é denso em $C^{\infty}\left(I, \mathbb{R}^{3}\right) \times\{\delta\}$ então

$$
T_{W,\left(t_{0}, u_{0}\right)}=\bigcap_{j=1}^{\infty} T_{W,\left(t_{0}, u_{0}\right), K_{j}}
$$

é residual.

Como $\operatorname{res}_{U_{3}}$ é sobrejetora é suficiente mostrar que

$$
T_{W,\left(t_{0}, u_{0}\right), K_{j}, U_{3}}=\left\{\gamma \in C^{\infty}\left(U_{3}, \mathbb{R}^{3}\right) / j_{1}^{k} \widetilde{\pi}_{3} \circ \widetilde{F}_{\gamma, \delta} \pitchfork W \operatorname{com} j_{1}^{k} \widetilde{\pi}_{3} \circ \widetilde{F}_{\gamma, \delta}\left(t_{0}, u_{0}\right) \in \bar{K}_{j}\right\}
$$

é denso em $C^{\infty}\left(U_{3}, \mathbb{R}^{3}\right)$.

Seja $P(1,2, k)$ o conjunto dos pares de polinômios $\left(p_{1}, p_{2}\right)$ de grau menor ou igual a $k$ sem termos constantes em uma variável.

Para qualquer que seja $\gamma \in C^{\infty}\left(U_{3}, \mathbb{R}^{3}\right)$ e $p=\left(p_{1}, p_{2}\right) \in P(1,2, k)$ definimos $f_{(\gamma, p)}$ : $U_{3} \times J \rightarrow \mathbb{R}^{2}$ por $f_{(\gamma, p)}(t, u)=\left(\gamma_{1}(t)+p_{1}(t)+g_{3}(t) \delta_{1}(t), \gamma_{2}(t)+p_{2}(t)+g_{3}(t) \delta_{2}(t)+u \delta_{2}(t)\right)$. 
Definimos, também, $\phi: U_{3} \times J \times P(1,2, k) \rightarrow J^{k}(1,2)$ por $\phi\left(t, u,\left(p_{1}, p_{2}\right)\right)=j_{1}^{k} f_{(\gamma, p)}(t, u)$ $=j^{k} f_{(\gamma, p), u}(t)$ onde $f_{(\gamma, p), u}=f_{(\gamma, p)}(t, u)$. Podemos considerar que $P(1,2, k)$ é o espaço euclideano $\mathbb{R}^{N}$.

Temos que $\phi$ é transversal a $W$ pois é submersão, assim pelo teorema de Transversalidade de Thom o conjunto $\left\{p \in P(1,2, k) / \phi_{p} \pitchfork W\right.$ em $\left.\left(t_{0}, u_{0}\right) \operatorname{com} \phi_{p}\left(t_{0}, u_{0}\right) \in \bar{K}_{j}\right\}$ é denso em $P(1,2, k)$.

Portanto podemos encontrar $p_{1}, \ldots, p_{k}, \ldots$ em $P(1,2, k)$ convergindo para $(0,0)$ tal que $\phi_{p_{i}} \pitchfork W$ sobre $K_{j}$. Como

$$
\lim _{i \rightarrow \infty}\left(\gamma+\left(p_{1, i}, p_{2, i}, 0\right)\right)=\gamma
$$

em $C^{\infty}\left(U_{3}, \mathbb{R}^{3}\right)$ então $T_{W,\left(t_{0}, u_{0}\right), K_{j}, U_{3}}$ é denso em $C^{\infty}\left(U_{3}, \mathbb{R}^{3}\right)$.

De maneira absolutamente análoga podemos proceder para $U_{1}$ e $U_{2}$.

Teorema 4.2.3 Existe $\mathcal{O} \subset C_{p r}^{\infty}\left(I, \mathbb{R}^{3} \times S^{2}\right)$ aberto e denso tal que o germe de superfície regrada $F_{(\gamma, \delta)}$ em todo ponto $\left(t_{0}, u_{0}\right)$ é um germe de imersão ou $\mathcal{A}$-equivalente ao guardachuva de Whitney para todo $(\gamma, \delta) \in \mathcal{O}$

Demonstração: Seja $K_{i}$ a $\mathcal{K}$-órbita com codimensão $i$ em $J^{k}(1,2)$ para $k$ suficientemente grande. Consideremos $\Sigma(1,2)=\cup_{i \geq 3} K_{i} \subset J^{k}(1,2)$. Temos que $\Sigma(1,2)$ é um subconjunto semi-algébrico de $J^{k}(1,2)$ com codimensão maior que 2.

Como $\Sigma(1,2)$ é semi-algébrico existe uma estratificação $\left\{\Im_{i}\right\}_{i=1}^{m}$ com codimensão $\Im_{i}>$ 2. Para todo $\left(t_{0}, u_{0}\right)$ com $\delta_{3}\left(t_{0}\right) \neq 0$ temos que $T_{\Sigma(1,2),\left(t_{0}, u_{0}\right)}=\bigcap_{i=1}^{m} T_{\Im_{i},\left(t_{0}, u_{0}\right)}$ pois se $f \pitchfork \Sigma(1,2)$ em $\left(t_{0}, u_{0}\right)$ então $f \pitchfork \Im_{i}$ em $\left(t_{0}, u_{0}\right)$ para todo $i$.

Pelo lema 4.2.2, $T_{K_{i},\left(t_{0}, u_{0}\right)}$ e $T_{\Sigma(1,2),\left(t_{0}, u_{0}\right)}$ são subconjuntos residuais de $C_{p r}^{\infty}\left(I, \mathbb{R}^{3} \times S^{2}\right)$ logo o conjunto

$$
\mathcal{O}_{\left(t_{0}, u_{0}\right)}=\cap_{i=1}^{2} T_{K_{i},\left(t_{0}, u_{0}\right)} \cap T_{\Sigma(1,2),\left(t_{0}, u_{0}\right)}
$$

é conjunto residual de $C_{p r}^{\infty}\left(I, \mathbb{R}^{3} \times S^{2}\right)$.

Podemos proceder de maneira análoga para $\delta_{j}\left(t_{0}\right) \neq 0(j=1,2)$, assim, usando o lema 4.1.17, para cada $\left(t_{0}, u_{0}\right) \in I \times J$ fixo, existe um subconjunto residual $\mathcal{O}_{\left(t_{0}, u_{0}\right)} \subset$ $C_{p r}^{\infty}\left(I, \mathbb{R}^{3} \times S^{2}\right)$, tal que o germe $F_{(\gamma, \delta)}$ em $\left(t_{0}, u_{0}\right)$ é infinitesimalmente $\mathcal{A}$-estável para todo $(\gamma, \delta) \in \mathcal{O}_{\left(t_{0}, u_{0}\right)}$.

Como um germe de $\mathbb{R}^{2} \rightarrow \mathbb{R}^{3}$ infinitesimalmente $\mathcal{A}$-estável é uma imersão (com cruzamento normal) ou um guarda-chuva de Whitney e as singularidades de $F_{(\gamma, \delta)}$ estão sobre a curva de estrição $(u=0)$, existe uma vizinhança $V_{t_{0}} \subset I$ de $t_{0}$ tal que $F_{(\gamma, \delta)}$ é 
uma imersão sobre $V_{t_{0}} \times J-\left\{\left(t_{0}, u_{0}\right)\right\}$. Como $\bar{I}$ é compacto podemos estender $(\gamma, \delta)$ para $I_{0} \supset \bar{I}$ e assim existe um numero finito de indices tais que $\cup_{i=1}^{l} V_{t_{i}}=I$.

Concluímos que $\mathcal{O}=\bigcap_{i=1}^{l} \mathcal{O}_{t_{0}, u_{0}}$ é subconjunto residual de $C_{p r}^{\infty}\left(I, \mathbb{R}^{3} \times S^{2}\right)$.

Mostremos, finalmente, que $\mathcal{O}$ é aberto. Consideremos a seguinte aplicação contínua $\digamma: C^{\infty}\left(I, \mathbb{R}^{3} \times S^{2}\right) \rightarrow C^{\infty}\left(I \times J, \mathbb{R}^{3}\right)$ definida por $\digamma(\gamma, \delta)=F_{(\gamma, \delta)}$.

Como o guarda-chuva de Whitney é a singularidade estável de germes de aplicação de $\left(\mathbb{R}^{2}, 0\right) \rightarrow\left(\mathbb{R}^{3}, 0\right)$ o conjunto

$\Gamma=\left\{f \in C^{\infty}\left(I \times J, \mathbb{R}^{3}\right) / f\right.$ é imersão ou guarda-chuva de Whitney, $\left.\forall(t, u) \in I \times J\right\}$

é aberto. Portanto $\mathcal{O}=\digamma^{-1}(\Gamma)$ é aberto. 


\section{Capítulo 5}

\section{Singularidades das Superfícies}

\section{Desenvolvíveis}

Nos capítulos anteriores mostramos que as singularidades genéricas que ocorrem no conjunto das superfícies regradas são as mesmas singularidades genéricas que ocorrem de maneira geral no conjunto das aplicações de $\mathbb{R}^{2}$ em $\mathbb{R}^{3}$. O que queremos agora é mostrar que num subconjunto das superfícies regradas, a saber o conjunto das superfícies desenvolvíveis, as singularidades genéricas que aparecem são mais degeneradas do que as que ocorrem de maneira geral no conjunto das superfícies regradas.

No capítulo 1 definimos superfície desenvolvível. Tais superfícies são formadas por partes de cones, cilindros e de superfícies tangentes a uma curva. Ou de maneira mais abrangente, se pudermos definir sua curva de estrição, então a superfície desenvolvível é a superfície tangente associada a esta curva que neste caso será dada pelo lugar geométrico das singularidades da própria superfície desenvolvível. Assim, é suficiente estudar as singularidades genéricas das superfícies tangentes. Este capítulo tem por base os trabalhos $[4,19,21,22,26]$.

\subsection{Singularidade das Superfícies Tangentes}

Definição 5.1.1 A superfície tangente associada a uma curva $\gamma: \mathbb{R} \rightarrow \mathbb{R}^{3}$ é a imagem da aplicação $\chi: \mathbb{R}^{2} \rightarrow \mathbb{R}^{3}$ dada por $\chi(t, u)=\gamma(t)+u \gamma^{\prime}(t)$.

Observação 5.1.2 De maneira geral tomamos $\gamma$ uma curva regular e parametrizada por comprimento de arco. Neste caso, em pontos da forma $\left(t_{0}, 0\right) \in \mathbb{R}^{2}$, o posto de $\chi$ é constante e igual a 1. 
Neste caso um tal germe $\chi$ não é finitamente determinado segundo [26]. Usando apenas esta observação como motivação vamos introduzir conceitos relativos da teoria de singularidades no conjunto dos germes que têm as mesmas propriedades que $\chi$.

Definição 5.1.3 Definimos por $C E$ o conjunto dos germes de aplicações de $\left(\mathbb{R}^{2}, 0\right)$ em $\left(\mathbb{R}^{3}, 0\right)$ que tem posto 1 em todos os pontos de uma curva diferenciável em $\left(\mathbb{R}^{2}, 0\right)$. Diremos que um germe $f \in C E$ é $k$-determinado em $C E$ se para todo germe $g \in C E$ com $j^{k} f=j^{k} g$ então $f$ e $g$ são $\mathcal{A}$-equivalentes.

Definição 5.1.4 Sejam $f \in C E$ e $F(x, u)=\left(f_{u}(x), u\right)$ um desdobramento de $f$ então $F$ é um $C E$-desdobramento de $f$ se existe um mergulho diferenciável de $\left(\mathbb{R} \times \mathbb{R}^{d}, 0\right)$ em $\left(\mathbb{R}^{2} \times \mathbb{R}^{d}\right)$ definido por $(t, u) \mapsto\left(s_{u}(t), u\right)$ onde $d f_{u}$ tem posto 1 em cada ponto de $s_{u}(t)$.

Definição 5.1.5 Um CE-desdobramento $F$ de $f$ é CE-versal se qualquer outro CEdesdobramento $G:\left(\mathbb{R}^{2} \times \mathbb{R}^{a}, 0\right) \rightarrow\left(\mathbb{R}^{3} \times \mathbb{R}^{a}\right)$ do mesmo germe $f$ é equivalente a um induzido por uma mudança de base, isto é, existe um germe diferenciável $h:\left(\mathbb{R}^{a}, 0\right) \rightarrow$ $\left(\mathbb{R}^{d}, 0\right)$ e uma família diferenciável de difeomorfismos $\Phi(x, u)=(\varphi(x, u), u)$ e $\Psi(X, u)=$ $(\psi(X, u), u)$ tal que $\Psi \circ G \circ \Phi(x, u)=\left(f_{h(u)}(x), u\right)$.

Definição 5.1.6 Um germe $f \in C E$ é CE-estável se ele é seu próprio desdobramento CE-versal.

Lema 5.1.7 Se $F: \mathbb{R}^{2} \times \mathbb{R}^{r} \rightarrow \mathbb{R}^{3} \times \mathbb{R}^{r}, F(x, y, u)=\left(x, y^{2}, y p_{u}\left(x, y^{2}\right), u\right)$ é um desdobramento versal do germe $f(x, y)=\left(x, y^{2}, y p\left(x, y^{2}\right)\right)$ então $\widetilde{F}(x, y, u)=\left(x, y^{2}, y^{3} p_{u}\left(x, y^{2}\right), u\right)$ é um desdobramento CE-versal do germe $\tilde{f}(x, y)=\left(x, y^{2}, y^{3} p\left(x, y^{2}\right)\right)$.

Demonstração: Seja $\widetilde{G}: \mathbb{R}^{2} \times \mathbb{R}^{s} \rightarrow \mathbb{R}^{3} \times \mathbb{R}^{s}$ um outro desdobramento de $\tilde{f}$ então podemos definir $G: \mathbb{R}^{2} \times \mathbb{R}^{s} \rightarrow \mathbb{R}^{3} \times \mathbb{R}^{s}$ como sendo um desdobramento de $f$ apenas substituindo $y^{3}$ por $y$ em $\widetilde{G}$.

Como $F$ é desdobramento versal de $f$ vale que existe $h: \mathbb{R}^{s}, 0 \rightarrow \mathbb{R}^{r}, 0$ tal que $G$ é equivalente a $F(x, y, h(v))$ assim por [20] existem difeomorfismos

$$
\varphi(x, y, v)=\left(r\left(x, y^{2}\right), y s\left(x, y^{2}\right), v\right)
$$

e

$$
\psi(X, Y, Z, V)=(r(X, Y), Y s(X, Y), Z t(X, Y), V)
$$

tais que $\psi \circ F(x, y, h(v))=G \circ \varphi(x, y, v)$. 
Definindo $\widetilde{\psi}(X, Y, Z, V)=\left(r(X, Y), Y s(X, Y), Z t(X, Y) s^{2}(X, Y), V\right)$, temos que $\widetilde{\psi} \circ$ $\widetilde{F}(x, y, h(v))=\widetilde{G} \circ \varphi(x, y, v)$.

Portanto $\widetilde{G}$ é equivalente a $\widetilde{F}(x, y, h(v))$. Logo, $\widetilde{F}$ é $C E$-versal.

Colorário 5.1.8 O germe $f(x, y)=\left(x, y^{2}, y^{3}\right)$ que é a imersão cuspidal e $\widehat{f}(x, y)=$ $\left(x, y^{2}, x y^{3}\right)$ que é o guarda-chuva de Whitney cuspidal são CE-estáveis.

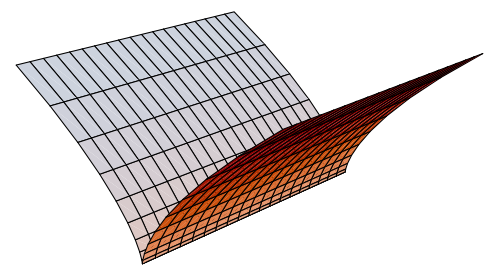

Figura 5.1: Imersão cuspidal

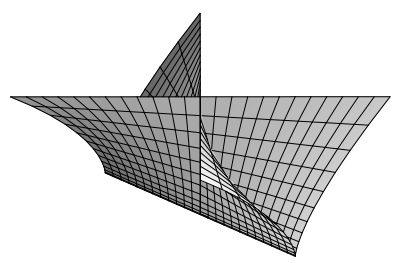

Figura 5.3: Guarda-chuva de Whitney cuspidal

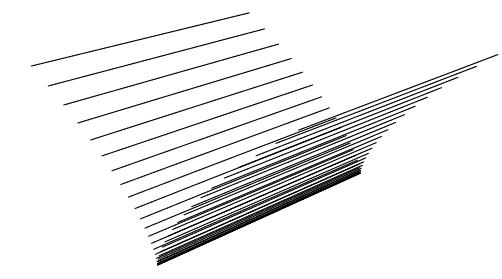

Figura 5.2: Imersão cuspidal regrado

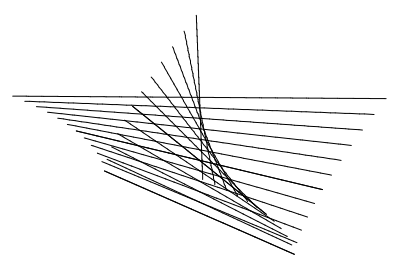

Figura 5.4: Guarda-chuva de Whitney cuspidal regrado

\subsection{Genericidade em Superfícies Tangentes}

Lema 5.2.1 Seja $\alpha \in \xi_{1,3}^{0}$ então

$$
W=\left\{j^{4} \alpha \in J^{4}(1,3) /\left\langle\alpha^{\prime}(0) \times \alpha^{\prime \prime}(0), \alpha^{\prime \prime \prime}(0)\right\rangle=\left\langle\alpha^{\prime}(0) \times \alpha^{\prime \prime}(0), \alpha^{(4)}(0)\right\rangle=0\right\}
$$

é fechado e é um subconjunto algébrico de codimensão 2 em $J^{4}(1,3)$.

Observação 5.2.2 Como todo conjunto algébrico tem uma estratificação, segundo [19], seja $\Im$ tal estratificação. 
Lema 5.2.3 Seja $G$ o conjunto das curvas regulares $\alpha \in C^{\infty}\left(\mathbb{R}, \mathbb{R}^{3}\right)$ tais que $j^{4} \alpha$ é transversal a $\Im$. $G$ é um conjunto aberto e denso de $C^{\infty}\left(\mathbb{R}, \mathbb{R}^{3}\right)$ e para todo $\alpha \in G$ e $t \in \mathbb{R}$ vale que $\left\langle\alpha^{\prime}(0) \times \alpha^{\prime \prime}(0), \alpha^{\prime \prime \prime}(0)\right\rangle \neq 0$ ou $\left\langle\alpha^{\prime}(0) \times \alpha^{\prime \prime}(0), \alpha^{(4)}(0)\right\rangle \neq 0$.

Demonstração: A demonstração segue do teorema da Transversalidade de Thom, bastando observar que $\operatorname{cod} W=2$.

Teorema 5.2.4 Seja $\alpha \in G$ e $t \in \mathbb{R}$. Então a curvatura $k(t)$ e a torção $\tau(t)$ de $\alpha$ estão bem definidas com $k(t) \neq 0$ e o germe, em $t$, da superfície tangente a $\alpha$ é $\mathcal{A}$-equivalente ao germe na origem de $f(x, y)=\left(x, y^{2}, y^{3}\right)$ se $\tau(t) \neq 0$ e $g(x, y)=\left(x, y^{2}, x y^{2}\right)$ se $\tau(t)=0$.

Demonstração: A torção e a curvatura em pontos da curva $\alpha$ são dados por

$$
\begin{gathered}
\tau(t)=\frac{\left(\alpha^{\prime}(t), \alpha^{\prime \prime}(t), \alpha^{\prime \prime \prime}(t)\right)}{\left\|\alpha^{\prime}(t) \times \alpha^{\prime \prime}(t)\right\|^{2}} \\
k(t)^{2}=\frac{\left\|\alpha^{\prime}(t) \times \alpha^{\prime \prime}(t)\right\|^{2}}{\left(\alpha^{\prime}(t) \cdot \alpha^{\prime}(t)\right)^{3}}
\end{gathered}
$$

Seja $\alpha \in G$ e $t \in \mathbb{R}$ então $\alpha^{\prime}(t) \times \alpha^{\prime \prime}(t) \neq 0$ ou $\alpha^{\prime}(t) \neq 0$. Nestas condições temos $k$ e $\tau$ bem definidas e $k(t) \neq 0$. Podemos supor que $\alpha$ é um representante do germe da curva $\alpha$ onde o ponto fixado é $t=0$.

Para finalizar o teorema basta mostrar que $T:\left(\mathbb{R}^{2}, 0\right) \rightarrow\left(\mathbb{R}^{3}, 0\right), T(t, u)=\alpha(t)+$ $u \alpha^{\prime}(t)$ é $\mathcal{A}$-equivalente a $f(x, y)=\left(x, y^{2}, y^{3}\right)$ se $\tau(0) \neq 0$ e a $f(x, y)=\left(x, y^{2}, x y^{3}\right)$ se $\tau(0)=0$.

Do lema anterior, temos que $\alpha^{\prime}(0) \neq 0$ e $\alpha^{\prime \prime}(0) \neq 0$. Podemos, então, reparametrizar $\alpha$ da seguinte forma: $\alpha(t)=(t, H(t), K(t))$ onde $H(t)=t^{2} \phi(t)$ e $K(t)=t^{3} \psi(t)$ se $\tau(0) \neq 0$ e $K(t)=t^{4} \psi(t)$ se $\tau=0$ tal que $\phi(0)=\psi(0)=1$. Logo $T$ é $\mathcal{A}$-equivalente a

$$
f_{0}(t, u)=\left(t+u, H(t)+u H^{\prime}(t), K(t)+u K^{\prime}(t)\right) .
$$

Fazendo a mudança de coordenadas $(x, y, z) \rightarrow\left(x_{1}, y_{1}, z_{1}\right)=(x, y-H(x), z-K(x))$ e a reparametrização $(t, u) \rightarrow(s, u)=(t+u, u)$ temos

$$
f_{1}(s, u)=\left(s, y_{1}(s, u), z_{1}(s, u)\right)
$$

onde $y_{1}(s, u)=H(s-u)+u H^{\prime}(s-u)-H(s)$ e $z_{1}(s, u)=K(s-u)+u K^{\prime}(s-u)-K(s)$.

Agora, existem germes $m$ e $n$ tais que

$$
y_{1}(s, u)=\frac{u^{2}}{2} H^{\prime \prime}(s)+\frac{u^{3}}{3} H^{\prime \prime \prime}(s)+u^{4} m(s, u) ;
$$




$$
z_{1}(s, u)=\frac{u^{2}}{2} K^{\prime \prime}(s)+\frac{u^{3}}{3} K^{\prime \prime \prime}(s)+u^{4} n(s, u) .
$$

Como, $H^{\prime \prime}(0)=2$, existe vizinhança da origem na qual $\frac{1}{H^{\prime \prime}}$ está bem definida. Então aplicando a mudança de coordenadas

$$
\left(x_{1}, y_{1}, z_{1}\right) \rightarrow\left(x_{2}, y_{2}, z_{2}\right)=\left(x_{1},-2 \frac{y_{1}}{H^{\prime \prime}\left(x_{1}\right)}, 3 z_{1}-3 \frac{y_{1} K^{\prime \prime}\left(x_{1}\right)}{H^{\prime \prime}\left(x_{1}\right)}\right)
$$

temos $f_{1} \sim f_{2}$ onde $f_{2}(s, u)=\left(s, u^{2}+u^{3} \widehat{m}(u, s), u^{3} D(s)+u^{4} \widehat{n}(s, u)\right)$, com $D(s)=$ $3\left(\frac{K^{\prime \prime \prime}(s)-H^{\prime \prime \prime}(s) K^{\prime \prime}(s)}{H^{\prime \prime}(s)}\right)$.

Reparametrizando por $(s, u) \rightarrow(x, y)=(x, u \sqrt{(1+u \widehat{m}(x, u))})$ concluímos que $f_{2} \sim$ $f_{3}$ onde $f_{3}(x, y)=\left(x, y^{2}, y^{3} D(x)+y^{4} \widetilde{k}(x, y)\right)$.

Pelo Teorema de Preparação de Malgrange (Teorema 4.1.13) existem germes $p$ e $q$ tais que $\widetilde{k}(x, y)=p\left(x, y^{2}\right)+y q\left(x, y^{2}\right)$. Portanto a mudança de coordenadas $\left(x_{2}, y_{2}, z_{2}\right) \rightarrow$ $\left(x_{3}, y_{3}, z_{3}\right)=\left(x_{2}, y_{2}, z_{2}-y_{2}^{2} p\left(x_{2}, y_{2}\right)\right)$ nos dá que $f_{3} \sim f_{4}$ onde $f_{4}\left(x, y^{2}, y^{3}\left(D(x)+y^{2} q\left(x, y^{2}\right)\right)\right)$.

Se $\tau(0) \neq 0, K(t)=t^{3} \psi(t)$. Então $D(0)=18$ e a mudança de coordenadas $\left(x_{3}, y_{3}, z_{3}\right) \rightarrow$ $\left(x_{3}, y_{3}, z_{3}\left(D\left(x_{3}\right)+y_{3} q\left(x_{3}, y_{3}\right)\right)^{-1}\right)$ nos dá $f_{4} \sim f$.

Por outro lado, se $\tau(0)=0, K(t)=t^{4} \psi(t)$. Então existe um germe $j$ tal que $D(x)=24 x+x^{2} j(x)$ e a mudança de coordenadas $\left(x_{3}, y_{3}, z_{3}\right) \rightarrow\left(D\left(x_{3}\right)+y_{3} q\left(x_{3}, y_{3}\right), y_{3}, z_{3}\right)$ juntamente com a reparametrização $\left(x^{\prime}, y\right)=\left(D(x)+y^{2} q\left(x, y^{2}\right), y\right)$ nos dá $f_{4} \sim g$.

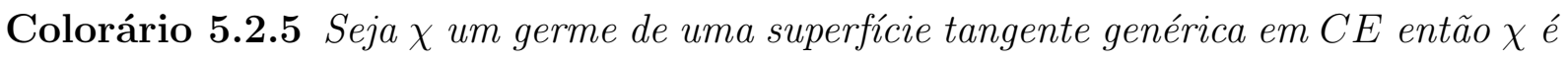
$\mathcal{A}$-equivalente a ao germe, na origem, de $f(x, y)=\left(x, y^{2}, y^{3}\right)$ ou de $g(x, y)=\left(x, y^{2}, x y^{2}\right)$.

Observação 5.2.6 A imersão cuspidal é a correspondente degenerada, no conjunto $C E$, à imersão no conjunto das superfícies regradas, enquanto o guarda-chuva de Whitney Cuspidal é o correspondente degenerado do guarda-chuva de Whitney. 


\section{Referências Bibliográficas}

[1] F.Apéry, Models of the Real Projective Plane, F.Vieweg and Sohn BraunSCHWEIG/Wiesbaden (1987).

[2] J.W. Bruce, A. du Plessis and C.T.C. Wall, Determinacy and Unipotency, InVENT. MATH., 88 (1987), 521-554.

[3] M. P. do Carmo, Differential Geometry of Curves and Surfaces, Prentice-Hall (1976).

[4] J. P. Cleave, The form of the tangent developable at points of zero torsion on space curves, Math. Proc. Camb. Phil. Soc., 88 (1980), 403-407.

[5] T. Farid, Singularidades de Aplicações Diferenciáveis, Notas Didáticas do ICMC-USP, 34 (1999).

[6] T. Gaffney, On the order of determination of a finitely determined germ., Invent. МАтн., 37 (1976), 83-92.

[7] C. Gibson, Singular points of smooth mappings, Pitman (1979).

[8] C.G. Gibson, K. Wirthmuller, A.A. du Plessis and E.J.N.Looijenga, Topological Stability of Smooth Mappings, Lecture Notes in Mathematicas, 552 (1976).

[9] M. Golubitsky e V. Gulllemin, Stability of smooth mappings and their singularities, Springer-Verlag (1973).

[10] J. Hoschek and H. Pottman, Interpolation and approximation with developable B-spline surfaces Mathematical Methods for Curves and surfaces (ed. M. Daehlen, T. Lyche and L. L. Schumacker), Vanderbilt University Press (1995), 255-264. 
[11] G. Ishikawa, Determinacy of envelope of the osculating hyperplanes to a curve, Bull. London Math. Soc., 25 (1993), 787-798.

[12] S. Izumiya, Singularities of ruled surfaces in $\mathbb{R}^{3}$, Mathematical Proceedings of the Cambridge Philosophical Society, 130 (2001), 1-11.

[13] S. Izumiya and N. Takeuchi, Special curves and ruled surfaces, Hokkaido Univer. Preprint Series in Math, (2000).

[14] S. Izumiya and N. Takeuchi, New special curves and developable surfaces, Hokkaido Univer. Preprint Series in Math, 555 (2002).

[15] J. Martinet, Singularities of smooth functions and maps, London MathematiCal Society Lecture Note Series, 58 (1961).

[16] J. N. Mather, Stability of $C^{\infty}$ mappings. I. The division theorem, Ann. of Math., 2 (1968), 89-104.

[17] J. N. Mather, Stability of $C^{\infty}$ mappings. II. Infinitesimal stability implies stability, AnN. OF MATH., 2 (1969), 254-291.

[18] J. N. Mather, Stability of $C^{\infty}$ mappings. III. Finitely determined mapgerms, Inst. Hautes Études Sci. Publ. Math., 35 (1968), 279-308.

[19] J. N. Mather, Stratifications and mappings, Dynamical Systems, ed. M.M. Peixoto, Academic Press, N.Y. (1973), 195-232.

[20] D. Mond, On the classification of germs of maps from $\mathbb{R}^{2}$ to $\mathbb{R}^{3}$, Proc. London Math. Soc., 50 (1985), 333-369.

[21] D. Mond, On the tangent developable of a space curve, Math. Proc. Camb. Phil. Soc., 91 (1981), 351-355.

[22] D. Mond, Singularities of the tangent developable surface of a space curve, QUART. J. Math. OxFord, 40 (1989), 79-91.

[23] A. Du Plessis, On the determinacy of smooth map-germs, Invent. Math., 58 (1980), 107-160.

[24] T. SASAKI, Projective differential geometry and linear homogeneous differential equations, Rokko Lectures in Math., Kobe University, 5 (1999). 
[25] M. SCHNeIDER, Interpolation with developable strip- surfaces consisting of cylinders and cones, in mathematical methods for curves and surfaces II, (ed. M. Daehlen T. Lyche and L. L. Schumacker), VAnderbilt University Press (1998), 437-444.

[26] C.T.C. Wall, Finite determinacy of smoth map-germs, Bull. London. Math. Soc., 13 (1981), 481-539.

[27] H. Whitney, The singularities of a smooth n-manifold in (2n-1)-space, ANNALS OF Math., 45 (1944), 247-293. 


\section{Índice Remissivo}

ação de grupo de Lie, 13

CE

conjunto, 40

desdobramento, 40

estável, 40

vesal, 40

co-posto, 11

cruzamento normal, 22

curva

base, 1

de estrição, 3

desdobramento, 27

equivalência, 29

induzido, 29

isomorfismo, 29

mini-versal, 29

morfismo, 29

versal, 29

determinação finita, 18

diretriz, 1

espaço de Baire, 12

estável, 14

infinitesimalmente, 15

estabilidade, 15

fibrado tangente, 15

função distribuição, 5

genericidade, 14 germe, 11

de difeomorfismo, 11

imersível, 11

infinitesimalmente estável, 18

submersível, 11

germes

$\mathcal{A}$-equivalentes, 17

$\mathcal{K}$-equivalentes, 17

grupo de Lie, 13

guarda-chuva de Whitney, 6, 18

cuspidal, 41

imersão cuspidal, 41

jatos, 11

pontos centrais, 3

posto, 11

residual, 12

semi-regular, 24

superfície

desenvolvível, 7, 39

regrada, 1

tangente, 2, 39

teorema

de preparação de Malgrange, 30

de transversalidade de Thom, 14

de Mather, 16

topologia de Whitney, 12 
transversal completa, 18

transversalidade, 13 